\title{
The CHESS survey of the L1157-B1 bow-shock: high and low excitation water vapor ${ }^{\star}$
}

\author{
G. Busquet ${ }^{1}$, B. Lefloch ${ }^{2,3}$, M. Benedettini ${ }^{1}$, C. Ceccarelli ${ }^{2}$, C. Codella ${ }^{4}$, S. Cabrit ${ }^{5}$, B. Nisini ${ }^{6}$, S. Viti ${ }^{7}$, \\ A. I. Gómez-Ruiz ${ }^{4}$, A. Gusdorf ${ }^{8}$, A. M. di Giorgio ${ }^{1}$, and L. Wiesenfeld ${ }^{2}$ \\ ${ }^{1}$ INAF - Istituto di Astrofisica e Planetologia Spaziali, via del Fosso del Cavaliere 100, 00133 Roma, Italy \\ e-mail: gemma.busquet@iaps.inaf.it \\ 2 UJF-Grenoble 1/CNRS-INSU, Institut de Planétologie et d'Astrophysique de Grenoble (IPAG) UMR 5274, 38041 Grenoble, France \\ 3 Centro de Astrobiologia, CSIC-INTA, Carretera de Torrejón a Ajalvir, km 4, Torrejón de Ardoz, 28850 Madrid, Spain \\ ${ }^{4}$ INAF - Osservatorio Astrofisico di Arcetri, Largo Enrico Fermi 5, 50125 Firenze, Italy \\ 5 Observatoire de Paris, LERMA, UMR 8112 du CNRS, ENS, UPMC, UCP, 61 Av. de l'Observatoire, 75014 Paris, France \\ 6 INAF - Osservatorio Astronomico di Roma, via di Frascati 33, 00040 Monte Porzio Catone, Italy \\ 7 Department of Physics and Astronomy, University College London, Gower Street, London WC1E 6BT, UK \\ 8 LERMA, UMR 8112 du CNRS, Observatoire de Paris, École Normale Supérieure, 24 rue Lhomond, 75231 Paris Cedex 05, France
}

Received 23 July 2013 / Accepted 12 November 2013

\section{ABSTRACT}

\begin{abstract}
Context. Molecular outflows powered by young protostars strongly affect the kinematics and chemistry of the natal molecular cloud through strong shocks. This results in substantial modifications of the abundance of several species. In particular, water is a powerful tracer of shocked material because of its sensitivity to both physical conditions and chemical processes.

Aims. As part of the Chemical HErschel Surveys of Star-forming regions (CHESS) guaranteed time key program, we aim at investigating the physical and chemical conditions of $\mathrm{H}_{2} \mathrm{O}$ in the brightest shock region $\mathrm{B} 1$ of the $\mathrm{L} 1157$ molecular outflow.

Methods. We observed several ortho- and para- $\mathrm{H}_{2} \mathrm{O}$ transitions using the HIFI and PACS instruments on board Herschel toward L1157-B1, providing a detailed picture of the kinematics and spatial distribution of the gas. We performed a large velocity gradient (LVG) analysis to derive the physical conditions of $\mathrm{H}_{2} \mathrm{O}$ shocked material, and ultimately obtain its abundance.

Results. We detected $13 \mathrm{H}_{2} \mathrm{O}$ lines with both instruments probing a wide range of excitation conditions. This is the largest data set of water lines observed in a protostellar shock and it provides both the kinematics and the spatial information of the emitting gas. The PACS maps reveal that $\mathrm{H}_{2} \mathrm{O}$ traces weak and extended emission associated with the outflow identified also with HIFI in the o- $\mathrm{H}_{2} \mathrm{O}$ line at $556.9 \mathrm{GHz}$, and a compact $\left(\sim 10^{\prime \prime}\right)$ bright, higher excitation region. The LVG analysis of $\mathrm{H}_{2} \mathrm{O}$ lines in the bowshock show the presence of two gas components with different excitation conditions: a warm $\left(T_{\text {kin }} \simeq 200-300 \mathrm{~K}\right)$ and dense $\left(n\left(\mathrm{H}_{2}\right) \simeq\right.$ $\left.(1-3) \times 10^{6} \mathrm{~cm}^{-3}\right)$ component with an assumed extent of $10^{\prime \prime}$, and a compact $\left(\sim 2^{\prime \prime}-5^{\prime \prime}\right)$ and hot, tenuous $\left(T_{\text {kin }} \simeq 900-1400 \mathrm{~K}, n\left(\mathrm{H}_{2}\right) \simeq\right.$ $10^{3-4} \mathrm{~cm}^{-3}$ ) gas component that is needed to account for the line fluxes of high $E_{\mathrm{u}}$ transitions. The fractional abundance of the warm and hot $\mathrm{H}_{2} \mathrm{O}$ gas components is estimated to be $(0.7-2) \times 10^{-6}$ and $(1-3) \times 10^{-4}$, respectively. Finally, we identified an additional component in absorption in the HIFI spectra of $\mathrm{H}_{2} \mathrm{O}$ lines that connect with the ground state level. This absorption probably arises from the photodesorption of icy mantles of a water-enriched layer at the edges of the cloud, driven by the external UV illumination of the interstellar radiation field.
\end{abstract}

Key words. stars: formation - ISM: individual objects: L1157-B1 - ISM: molecules - ISM: abundances - ISM: jets and outflows

\section{Introduction}

Molecular outflows are among the most conspicuous manifestations of a nascent star. These outflows are known to result from the entrainment of circumstellar gas, swept up by the primary jet, where a shock front is generated as a consequence of the supersonic impact of the jet with the natal cloud. Shocks heat, accelerate, and compress the ambient gas material switching on a complex chemistry that leads to an enhancement of the abundance of several species in the so-called chemically active outflows (e.g., Bachiller 1996). The nature and properties of these shocks are still not well understood, in particular the role of the magnetic field. Water is predicted to be one of the main gas cooling agents

* Based on Herschel HIFI and PACS observations. Herschel is an ESA space observatory with science instruments provided by European-led Principal Investigator consortia and with important participation from NASA. in magnetized shocks, along with $\mathrm{H}_{2}$ and $\mathrm{CO}$ (e.g., Draine et al. 1983; Kaufman \& Neufeld 1996; Flower \& Pineau des Forêts 2010). Thanks to its rich emission spectrum, transitions spanning a wide range of excitation conditions, and its sensitivity to local conditions (e.g., Bergin et al. 1998; van Dishoeck et al. 2011), $\mathrm{H}_{2} \mathrm{O}$ constitutes a powerful probe of the physics and chemistry of the shock outflow interaction. In particular, in shocked regions $\mathrm{H}_{2} \mathrm{O}$ abundance can increase by several orders of magnitude, up to $\sim 10^{-4}$, through sputtering of grains mantles and formation in the gas phase at high temperatures (Hollenbach \& McKee 1989; Kaufman \& Neufeld 1996; Flower \& Pineau des Forêts 2010).

The outflow powered by the low-mass Class 0 protostar L1157-mm ( $d \simeq 250$ pc; Looney et al. 2007) displays a rich specific chemistry that makes it the prototype of chemically active outflows (e.g., Bachiller \& Pérez Gutiérrez 1997; Bachiller et al. 2001; Arce et al. 2008). As such, it is an excellent laboratory for investigating the physical conditions and the formation 


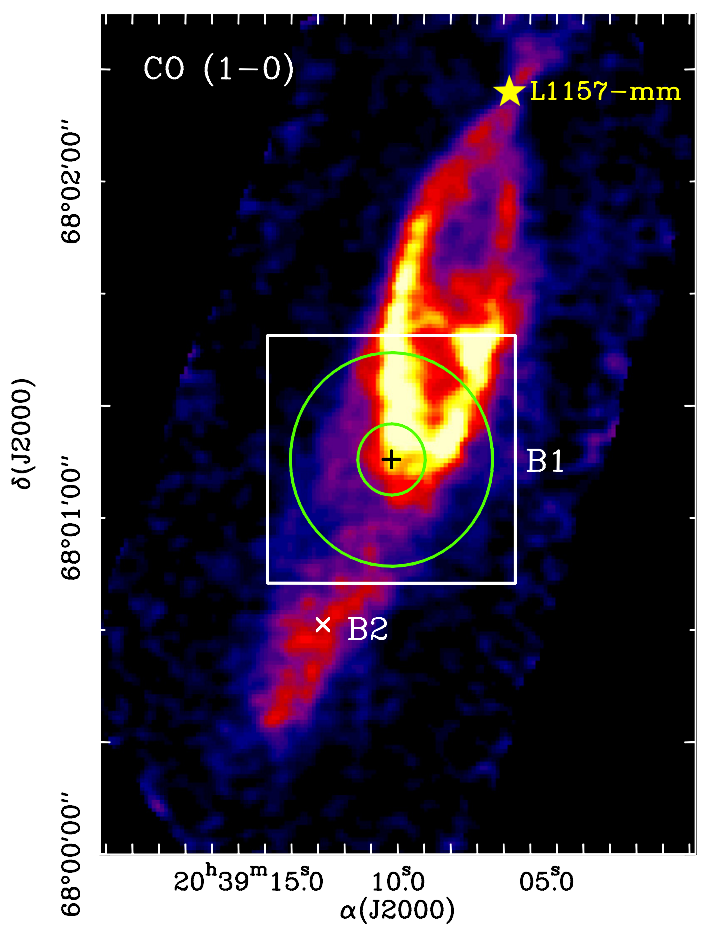

Fig. 1. Southern blueshifted outflow lobe observed in $\mathrm{CO}(1-0)$ with the Plateau de Bure Interferometer (PdBI) by Gueth et al. (1996). The black cross marks the nominal position of the bow-shock L1157-B1 while the white $\times$ symbol indicates the nominal position of the B2 shock (Bachiller \& Pérez Gutiérrez 1997). The largest $\left(\sim 38^{\prime \prime}\right)$ and smallest $\left(\sim 12^{\prime \prime} .7\right)$ HIFI main-beams are indicated with circles. The field of view of the PACS observations is displayed with a white box. The star depicts the position of the protostar L1157-mm.

routes of $\mathrm{H}_{2} \mathrm{O}$ and its role in the cooling of a typical protostellar outflow. The L1157 outflow has been studied in detail for more than two decades through many molecular lines and in a wide range of wavelengths, from the near infrared (near-IR; e.g., Davis \& Eisloeffel 1995; Neufeld et al. 2009; Nisini et al. 2010 b) to the radio domain (e.g., Zhang et al. 2000; Bachiller et al. 2001; Tafalla \& Bachiller 1995). Several compact shocked regions are found along both the blue- and redshifted lobes (see e.g., Gueth et al. 1998; Nisini et al. 2007, 2010b). In particular, the southern blueshifted lobe, shown in Fig. 1, consists of two limb-brightened cavities, each of which is associated with a bow-shock, probably created by episodic events in a precessing jet (Gueth et al. 1996).

Water emission in L1157-B1 was first detected with the Infrared Space Observatory (ISO) by Giannini et al. (2001); however, only three lines were detected and the physical conditions of $\mathrm{H}_{2} \mathrm{O}$ could not be constrained. Later on, Odin and the Submillimeter Wave Astronomy Satelilte (SWAS) observed the fundamental $\mathrm{o}-\mathrm{H}_{2} \mathrm{O}$ line emission in the direction of the southern blueshifted lobe of the outflow (Bjerkeli et al. 2009; Franklin et al. 2008). The low angular resolution gave access only to properties averaged over the entire outflow lobe. Assuming that both the $\mathrm{H}_{2} \mathrm{O}$ and the low- $J \mathrm{CO}$ line emission originate in the same gas, these authors inferred an $\mathrm{o}-\mathrm{H}_{2} \mathrm{O}$ abundance ranging between $10^{-6}$ and $2 \times 10^{-4}$. As part of the Water In Star-forming regions with Herschel (WISH) key program, Nisini et al. (2010a) used PACS to map the o- $\mathrm{H}_{2} \mathrm{O} 179 \mu \mathrm{m}$ line over the entire outflow structure. These authors detected extended emission, with several strong peaks associated with shocked knots, well spatially correlated with $\mathrm{H}_{2}$ rotational lines (Nisini et al. 2010b).
The molecular bright shock region B1, in the southern lobe of the outflow (see Fig. 1), was selected as one of the targets of the key program Chemical HErschel Surveys of Star-forming regions $\left(\mathrm{CHESS}^{1}\right)$ dedicated to unbiased spectral line surveys of prototypical star-forming regions (Ceccarelli et al. 2010) in the guaranteed time of the Herschel Space Observatory (Pilbratt et al. 2010).

The CHESS survey of L1157-B1 offers a comprehensive view on the water line emission in a typical protostellar bowshock, considered the benchmark for shock models (Gusdorf et al. 2008a,b; Flower \& Pineau des Forêts 2010, 2012). A grand total of 13 water lines (both ortho and para) have been detected across the submillimeter and far-infrared window with the PACS spectro-imager (Poglitsch et al. 2010) and the HIFI heterodyne instrument (de Graauw et al. 2010), the largest data set of water lines detected so far in a protostellar shock. Both instruments provide us with a detailed picture of the kinematics and the spatial distribution of the water emission in L1157-B1, allowing us to derive strong constraints on the water abundance and the physical conditions in the emitting gas. The layout of the paper is as follows. In Sect. 2 we summarize our observations. In Sect. 3 we present the main results of HIFI and PACS and in Sect. 4 we analyze the excitation conditions of $\mathrm{H}_{2} \mathrm{O}$ using a large velocity gradient (LVG) model and discuss the origin of the water emission in L1157-B1, presenting, for the first time, a detailed picture of the bow-shock structure through Herschel observations of water lines. Finally, in Sect. 5 we list the main conclusions.

\section{Observations and data reduction}

\subsection{HIFI observations}

The HIFI observations were performed in double beam switching mode during 2010 towards the nominal position of B1: $\alpha(J 2000)=20^{\mathrm{h}} 39^{\mathrm{m}} 10^{\mathrm{s}} .2, \delta(J 2000)=68^{\circ} 01^{\prime} 10^{\prime} .5$. Both polarizations ( $\mathrm{H}$ and $\mathrm{V}$ ) were observed simultaneously. The receiver was tuned in double sideband (DSB). Most of the submillimeter window was covered in an unbiased way with HIFI, and the observations were carried out in spectral scanning mode. In order to study the properties of the $\mathrm{H}_{2} \mathrm{O}$ gas in the high-velocity wings of the outflow, a few lines were observed in pointed mode in order to reach an excellent signal-to-noise ratio $(\mathrm{S} / \mathrm{N})$.

We used the wide band spectrometer (WBS), which provides a frequency resolution of $0.5 \mathrm{MHz}$ (i.e., velocity resolution between $0.1 \mathrm{~km} \mathrm{~s}^{-1}$ and $0.4 \mathrm{~km} \mathrm{~s}^{-1}$, depending on the wavelength). The data were processed with the ESA supported package Herschel interactive processing environment ${ }^{2}$ (HIPE, Ott 2010) version 6 . Then level 2 fits files were exported and transformed into the GILDAS ${ }^{3}$ format for baseline subtraction and subsequent sideband deconvolution, which was performed manually. The relative calibration between both receivers $(\mathrm{H}$ and $\mathrm{V}$ ) was found to be very good, and both signals were co-added to improve the noise rms of the data.

The spectral resolution was then degraded to a common velocity resolution of $1 \mathrm{~km} \mathrm{~s}^{-1}$ in the final single side band (SSB) data set. Uncertainty in the flux calibration was estimated to be $\sim 20 \%$. In Table 1 we summarize the observational parameters of the $\mathrm{H}_{2} \mathrm{O}$ transitions detected with HIFI (frequency,

\footnotetext{
http://www-laog.obs.ujf-grenoble.fr/heberges/chess

2 HIPE is a joint development by the Herschel Science Ground Segment Consortium, consisting of ESA, the NASA Herschel Science Center, and the HIFI, PACS, and SPIRE consortia.

3 See http://www.iram. fr/IRAMFR/GILDAS
} 
Table 1. List of $\mathrm{H}_{2} \mathrm{O}$ transitions ${ }^{a}$ observed with HIFI in L1157-B1.

\begin{tabular}{|c|c|c|c|c|c|c|c|c|c|c|}
\hline Transition & $\begin{array}{c}\text { Frequency } \\
(\mathrm{GHz})\end{array}$ & $\begin{array}{c}\lambda \\
(\mu \mathrm{m})\end{array}$ & $\begin{array}{c}E_{\mathrm{u}} / k_{\mathrm{B}} \\
(\mathrm{K})\end{array}$ & $\begin{array}{c}H P B W \\
(\operatorname{arcsec})\end{array}$ & $\eta_{\mathrm{mb}}$ & Obs_Id & $\begin{array}{l}\mathrm{rms}^{b} \\
(\mathrm{mK})\end{array}$ & $\begin{array}{c}T_{\text {peak }} \\
(\mathrm{K})\end{array}$ & $\begin{array}{c}v_{\text {peak }} \\
\left(\mathrm{km} \mathrm{s}^{-1}\right)\end{array}$ & $\int_{\left(\mathrm{K} \mathrm{km} \mathrm{s}^{-1}\right)} T_{\mathrm{mb}} \mathrm{d} v$ \\
\hline $\mathrm{o}-\mathrm{H}_{2} \mathrm{O} 1_{10}-1_{01}$ & 556.936 & 538.66 & 26.7 & 38.1 & 0.75 & 1342181160 & 8 & $1.04(0.16)$ & $-1.9(0.3)$ & $13.0(0.1)$ \\
\hline $3_{12}-3_{03}$ & 1097.365 & 273.38 & 215.1 & 19.3 & 0.74 & 1342196453 & 26 & $0.29(0.04)$ & $-2.0(0.3)$ & $4.1(0.2)$ \\
\hline $3_{12}-2_{21}$ & 1153.127 & 260.17 & 215.1 & 18.3 & 0.64 & 1342207691 & 39 & $0.18(0.03)$ & $-1.9(0.1)$ & $1.3(0.3)$ \\
\hline $3_{21}-3_{12}$ & 1162.912 & 257.98 & 271.0 & 18.3 & 0.64 & 1342207691 & $36^{c}$ & $0.11(0.03)$ & $-1.7(0.4)$ & $0.5(0.2)$ \\
\hline $2_{21}-2_{12}$ & 1661.008 & 180.49 & 159.8 & 12.7 & 0.71 & 1342196538 & 138 & $0.36(0.14)$ & $-1.7(0.1)$ & $2.0(0.8)$ \\
\hline $2{ }_{12}-1_{01}$ & 1669.905 & 179.52 & 80.1 & 12.7 & 0.71 & 1342207689 & 56 & $0.94(0.14)$ & $-5.5(0.3)$ & $12.7(0.4)$ \\
\hline $\mathrm{p}-\mathrm{H}_{2} \mathrm{O} 2_{11}-2_{02}$ & 752.033 & 398.92 & 136.9 & 28.2 & 0.75 & 1342207611 & 20 & $0.46(0.07)$ & $-3.6(0.3)$ & $4.6(0.1)$ \\
\hline $2_{02}-1_{11}$ & 987.927 & 303.67 & 100.8 & 21.5 & 0.74 & 1342207640 & 50 & $0.79(0.12)$ & $-3.3(0.2)$ & $11.0(0.3)$ \\
\hline $1_{11}-0_{00}$ & 1113.343 & 269.47 & 53.4 & 19.1 & 0.74 & 1342207388 & 56 & $0.79(0.12)$ & $-4.1(0.1)$ & $11.1(0.4)$ \\
\hline
\end{tabular}

Notes. Peak intensity (in $T_{\mathrm{mb}}$ scale), peak velocity, and integrated intensity between -40 and $+2.6 \mathrm{~km} \mathrm{~s}^{-1}$ are reported. The uncertainties are indicated in parentheses. ${ }^{(a)}$ Frequencies taken from the JPL spectroscopic catalog (Pickett et al. 1998). ${ }^{(b)}$ Root mean square (rms) noise is given for an interval of $0.5 \mathrm{MHz} .{ }^{(c)}$ Root mean square (rms) noise is given for an interval of $1.5 \mathrm{MHz}$.

Table 2. List of $\mathrm{H}_{2} \mathrm{O}$ transitions ${ }^{a}$ detected with PACS in L1157-B1, at the nominal position of B1 (spaxel centered at offset $\left(0^{\prime \prime}, 0^{\prime \prime}\right)$ ) and at the high- $J$ CO peak (spaxel at offset $\left(-5^{\prime \prime}, 7^{\prime \prime}\right)$ ).

\begin{tabular}{|c|c|c|c|c|c|c|c|}
\hline \multirow[t]{2}{*}{ Transition } & \multirow{2}{*}{$\begin{array}{l}\text { Frequency } \\
\qquad(\mathrm{GHz})\end{array}$} & \multirow{2}{*}{$\begin{array}{c}\lambda \\
(\mu \mathrm{m})\end{array}$} & \multirow{2}{*}{$\begin{array}{c}E_{\mathrm{u}} / k_{\mathrm{B}} \\
(\mathrm{K})\end{array}$} & \multirow{2}{*}{$\begin{array}{l}H P B W \\
(\operatorname{arcsec})\end{array}$} & \multicolumn{2}{|c|}{ Flux $^{b}$} & \multirow[t]{2}{*}{ Line_Id } \\
\hline & & & & & $\begin{array}{r}\left(\times 10^{-17}\right) \\
\text { offset }\left(0^{\prime \prime}, 0^{\prime \prime}\right)\end{array}$ & $\begin{array}{l}\left.\mathrm{m}^{-2} / \text { pixel }\right) \\
\text { offset }\left(-5^{\prime \prime}, 7^{\prime \prime}\right)\end{array}$ & \\
\hline $\mathrm{o}-\mathrm{H}_{2} \mathrm{O} 2_{21}-2_{12}$ & 1661.008 & 180.49 & 159.8 & 12.7 & $2.6 \pm 0.2$ & $2.7 \pm 0.3$ & 1 \\
\hline $2_{12}-1_{01}$ & 1669.905 & 179.52 & 80.1 & 12.7 & $12.1 \pm 0.2$ & $12.9 \pm 0.3$ & 2 \\
\hline $3_{03}-2_{12}$ & 1716.769 & 174.63 & 162.5 & 12.3 & $6.2 \pm 0.3$ & $6.5 \pm 0.3$ & 3 \\
\hline $4_{23}-4_{14}$ & 2264.149 & 132.41 & 397.9 & 9.4 & $<0.6^{c}$ & $0.6 \pm 0.2$ & 4 \\
\hline $4_{14}-3_{03}^{d}$ & 2640.474 & 113.54 & 289.2 & 8.0 & $2.8 \pm 0.5$ & $3.7 \pm 0.4$ & 5 \\
\hline $2_{21}-1_{10}$ & 2773.978 & 108.07 & 159.8 & 7.6 & $3.2 \pm 0.4$ & $3.9 \pm 0.5$ & 6 \\
\hline $3_{21}-2_{12}$ & 3977.046 & 75.38 & 271.0 & 5.3 & $<2.1^{c}$ & $1.9 \pm 0.7$ & 7 \\
\hline $\mathrm{p}-\mathrm{H}_{2} \mathrm{O} 3_{13}-2_{02}$ & 2164.132 & 138.53 & 204.7 & 9.8 & $2.7 \pm 0.3$ & $2.7 \pm 0.3$ & 8 \\
\hline $4_{04}-3_{13}$ & 2391.573 & 125.35 & 319.5 & 8.9 & $1.1 \pm 0.3$ & $1.4 \pm 0.3$ & 9 \\
\hline
\end{tabular}

Notes. ${ }^{(a)}$ Frequencies taken from the JPL spectroscopic catalog (Pickett et al. 1998). ${ }^{(b)}$ Line fluxes obtained with a Gaussian fit of the line profile. (c) $3 \sigma$ upper limit based on the rms estimated at offset $\left(-5^{\prime \prime}, 7^{\prime \prime}\right) .{ }^{(d)}$ This line is blended with the $\mathrm{CO}(23-22)$ transition. To estimate the flux of the water line we subtracted the flux of $\mathrm{CO}(23-22)$ transition predicted by the model presented in Benedettini et al. (2012).

wavelength, and upper level energy). The main-beam intensity, peak velocity, and the integrated intensity for each transition are also reported. Intensities are expressed in units of main-beam brightness temperature. The telescope parameters (half power beamwidth (HPBW) and main-beam efficiency $\left.\left(\eta_{\mathrm{mb}}\right)\right)$ are taken from Roelfsema et al. (2012).

\subsection{PACS observations}

The PACS observations were carried out on May 25, 2010 in line spectroscopy mode in order to obtain a full-range spectrum of the molecular line emission towards B1, from 55-95.2 $\mu \mathrm{m}$ and from 101.2-210 $\mu \mathrm{m}$. The spectral scan was centered at the nominal position of B1 (see above) and produced a single $5 \times 5$ spectral map of 9.' 4 square spatial pixels (hereafter spaxels) over a $47^{\prime \prime} \times 47^{\prime \prime}$ field of view. Two observations were conducted for the 161.5-190.2 $\mu \mathrm{m}$ range. Both measurements are in good agreement, with a discrepancy of at the most $\sim 10 \%$. The resolving power ranges from 1000 to 4000 (i.e., spectral resolution of $\sim 75-300 \mathrm{~km} \mathrm{~s}^{-1}$ ) depending on the wavelength, hence water lines are unresolved. The PACS data were processed with HIPE version 5.0. The absolute flux scale was determined from observations of Neptune by normalizing the observed flux to the telescope background, with an estimated uncertainty of $\sim 10 \%$ for $\lambda<190 \mu \mathrm{m}$ (i.e., where all the water PACS lines lie). Further details of the PACS observations are described in Benedettini et al. (2012), where the emission lines of $\mathrm{CO}, \mathrm{OH}$, and the [OI] lines are presented and discussed.

In Table 2 we list the detected transitions, giving their frequency, wavelength, upper energy level, and beam size. We extracted the flux toward each spaxel adopting a Gaussian instrumental response. The line fluxes measured toward the two brightest spaxels, at offsets $\left(0^{\prime \prime}, 0^{\prime \prime}\right)$ and $\left(-5^{\prime \prime}, 7^{\prime \prime}\right)$, associated with the nominal position of B1 and the high-excitation CO emission peak (Benedettini et al. 2012), respectively, are given in Table 2.

\subsection{Cross calibration}

Four lines were observed by both PACS and HIFI instruments, which allowed us to check the consistency of the calibration: $\mathrm{H}_{2} \mathrm{O}\left(2_{12}-1_{01}\right)$ at $179 \mu \mathrm{m},\left(2_{21}-2_{12}\right)$ at $180 \mu \mathrm{m}$, $\mathrm{CO}(16-15)$, and $\mathrm{CO}(14-13)$. Using the PACS maps, we estimated the line intensities in the HIFI main-beam solid-angle towards the nominal position of B1. Comparison of the HIFIand PACS-based line intensities shows a very good agreement for $\mathrm{H}_{2} \mathrm{O}\left(2_{12}-1_{01}\right)$ and $\mathrm{CO}(14-13)$, where the integrated intensities are $13.7 / 14.2 \mathrm{~K} \mathrm{~km} \mathrm{~s}^{-1}$ and $5.0 / 5.5 \mathrm{~K} \mathrm{~km} \mathrm{~s}^{-1}$, respectively (for HIFI/PACS instruments), resulting in a discrepancy of $\sim 10 \%$. For the $\mathrm{H}_{2} \mathrm{O}\left(2_{21}-2_{12}\right)$ and $\mathrm{CO}(16-15)$ lines, 


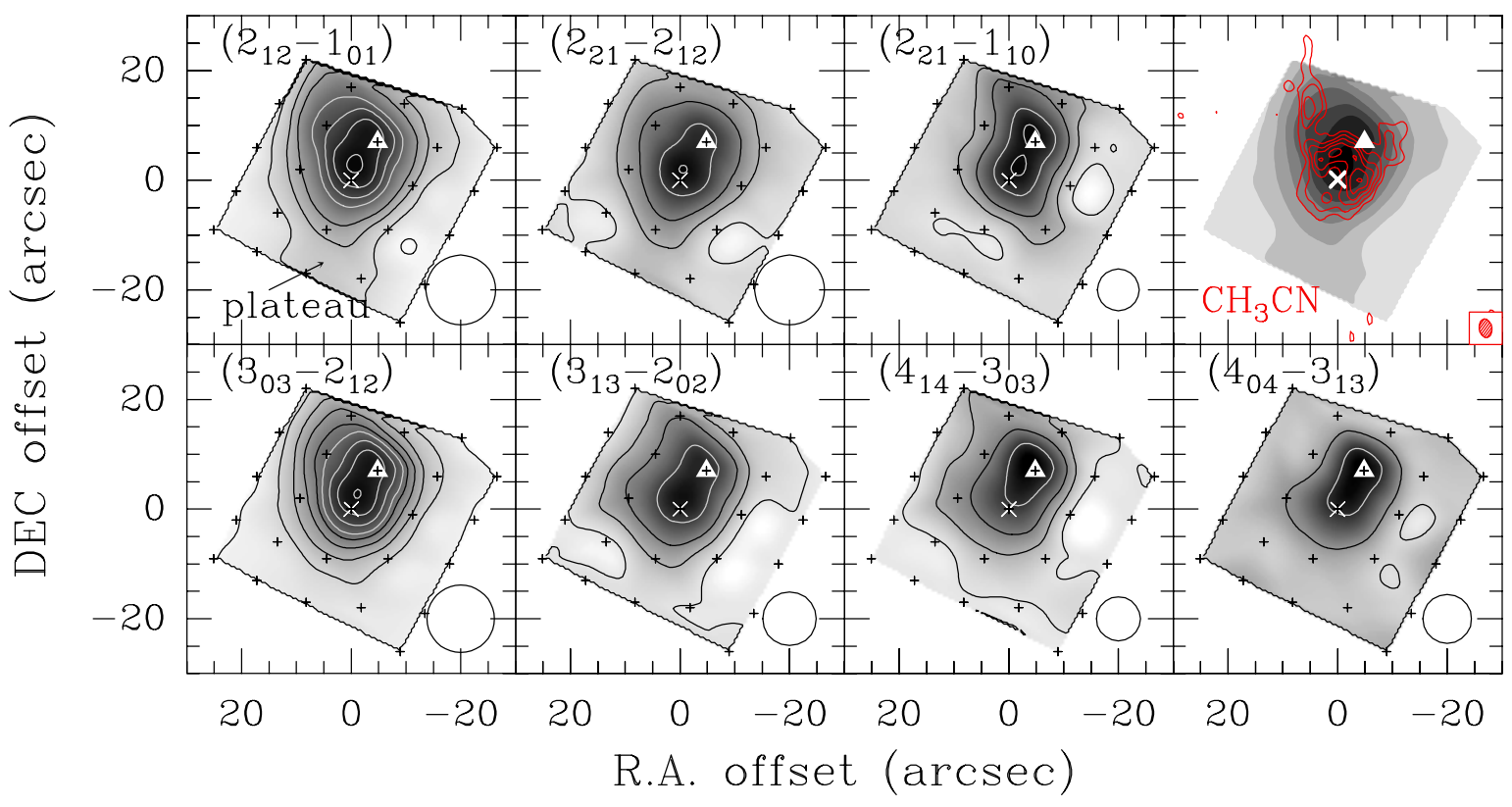

Fig. 2. PACS maps of $\mathrm{H}_{2} \mathrm{O}$ line fluxes (increasing upper-level energy from top left to bottom right). First contour corresponds to the $1 \sigma$ flux level of each transition. The contour step is $2 \sigma$ in all lines except for the $\mathrm{o}-\mathrm{H}_{2} \mathrm{O}\left(2_{12}-1_{01}\right)$ and $\mathrm{o}-\mathrm{H}_{2} \mathrm{O}\left(3_{03}-2_{12}\right)$, for which the contour step is $6 \sigma$ and $3 \sigma$, respectively, where $\sigma$ is listed in Table 2. The top right panel shows an overlay of the $\mathrm{o}-\mathrm{H}_{2} \mathrm{O}\left(2_{12}-1_{01}\right)$ map (gray scale) with the PdBI $\mathrm{CH}_{3} \mathrm{CN}(8-7) K=0-2$ image (red contours) from Codella et al. (2009) tracing the bow-shock. The synthesized beam of PdBI, 3'. $4 \times 2$ '. 3 $\left(\mathrm{PA}=10^{\circ}\right)$, is shown in the bottom right corner of this panel. Plus signs mark the central position of each spaxel of the PACS field of view. The white triangle indicates the position of the CO peak traced by high- $J_{\text {up }}$ PACS lines (Benedettini et al. 2012) and the $\times$ symbol depicts the nominal position of the B1 shock. The half power beam width (HPBW) of each transition is indicated in the bottom right corner of each panel and is listed in Table 2.

the HIFI/PACS integrated intensities are $2.0 / 1.8 \mathrm{~K} \mathrm{~km} \mathrm{~s}^{-1}$ and $3.4 / 2.7 \mathrm{~K} \mathrm{~km} \mathrm{~s}^{-1}$, respectively. For these lines the discrepancy is larger, about $\sim 20 \%$, but always within the absolute flux calibration uncertainty. We note that both lines are weaker, and the $\mathrm{S} / \mathrm{N}$ of the data much lower than the other two lines. Overall, we conclude that the agreement between the HIFI and PACS calibration scales is very good.

\section{Results}

We have detected $13 \quad \mathrm{H}_{2} \mathrm{O}$ transitions with a flux above the $5 \sigma$ level: $7 \mathrm{H}_{2} \mathrm{O}$ transitions (5 ortho, 2 para) in the PACS range $(55-210 \mu \mathrm{m}), 8 \mathrm{H}_{2} \mathrm{O}$ transitions (5 ortho, 3 para) with HIFI between $672 \mu \mathrm{m}$ and $180 \mu \mathrm{m}$. We note that the $179 \mu \mathrm{m}$ and $180 \mu \mathrm{m}$ lines have been detected with both instruments. The o- $\mathrm{H}_{2} \mathrm{O}\left(3_{21}-3_{12}\right)$ transition is detected at a $3 \sigma$ level with HIFI (see Table 1). Two additional o- $\mathrm{H}_{2} \mathrm{O}$ transitions $\left(3_{21}-2_{10}\right.$ at $75.38 \mu \mathrm{m}$ and $4_{23}-4_{14}$ at $132.41 \mu \mathrm{m}$ ) are tentatively detected with PACS at the $3 \sigma$ level at the offset position $\left(-5^{\prime \prime}, 7^{\prime \prime}\right.$ ) (see Table 2). Overall, we detected only transitions of rather low $E_{\mathrm{u}}$, with values ranging between $26.7 \mathrm{~K}$ and $319.5 \mathrm{~K}$.

\section{1. $\mathrm{H}_{2} \mathrm{O}$ spatial distribution}

Maps of $\mathrm{o}-\mathrm{H}_{2} \mathrm{O}$ and $\mathrm{p}-\mathrm{H}_{2} \mathrm{O}$ lines observed with PACS are presented in Fig. 2. Water is detected over the entire outflow cavity, both upstream and downstream of the bow-shock; its distribution overlaps rather well with the B1 bow-shock as traced by $\mathrm{CH}_{3} \mathrm{CN}$ (Codella et al. 2009, see top right panel of Fig. 2) and the outflow walls of the B1 cavity, traced by $\mathrm{CO}$ at the PdBI (Gueth et al. 1996, see Fig. 1). Downstream of B1, weak emission is present $20^{\prime \prime}$ away from the shock in several transitions, including the $3_{03}-2_{12}$ and $2_{12}-1_{01}$ lines, in agreement with
Nisini et al. (2010a). This extended emission, which consists of a plateau of low $\mathrm{H}_{2} \mathrm{O}$ brightness, is related to the ouflow, possibly from the $\mathrm{B} 2$ outflow cavity.

Leaving aside the contribution of the plateau to the emission, the distribution of the water emission in B1 displays little variation between the various transitions, with a typical deconvolved size at full-width at half maximum (FWHM) of $\sim 10^{\prime \prime}$. Overall, the emission appears elongated along the major outflow axis. The $\mathrm{H}_{2} \mathrm{O}$ brightness peak is located $\approx 6^{\prime \prime}$ north of the center of the PACS array, lying approximately halfway between the nominal position of $\mathrm{B} 1$ and the high- $J \mathrm{CO}$ emission peak identified by Benedettini et al. (2012), at the interface between spaxels $(2,2)$ and $(3,2)$ at offsets $\left(0^{\prime \prime}, 0^{\prime \prime}\right)$ and $\left(-5^{\prime \prime}, 7^{\prime \prime}\right)$, respectively. However, size and position determination from the PACS undersampled data are just indicative and they suffer large uncertainties.

It is interesting to compare the morphology of the $2_{12}-1_{01}$ line with that of other shock tracers (see Fig. 3). One can see that both $\mathrm{H}_{2} \mathrm{O}$ and $\mathrm{SiO}$ peak at the same position, between offset $\left(0^{\prime \prime}, 0^{\prime \prime}\right)$ and $\left(-5^{\prime \prime}, 7^{\prime \prime}\right)$. Similar to $\mathrm{H}_{2} \mathrm{O}$, the emission of the mid-IR $\mathrm{H}_{2} \mathrm{O}-0 \mathrm{~S}(1)$ pure-rotational line observed with Spitzer (Nisini et al. 2010b) is extended, partly tracing the B1 cavity, while $\mathrm{CO}(16-15)$ and the near-IR $\mathrm{H}_{2} 1-0 \mathrm{~S}(1)$ rovibrational line (Caratti o Garatti et al. 2006) are rather compact and peak at offset $\left(-5^{\prime \prime}, 7^{\prime \prime}\right)$, coinciding with the partly dissociative shock driven by the impact of the jet against the B1 cavity (Benedettini et al. 2012). However, it is worth noting that there is also bright $\mathrm{H}_{2} \mathrm{O}$ emission at the peak of the high- $J \mathrm{CO}$ position, suggesting that part of the $\mathrm{H}_{2} \mathrm{O}$ emission coincides with $\mathrm{CO}$. The good match between $\mathrm{SiO}(2-1)$ and $\mathrm{H}_{2} \mathrm{O}\left(2_{12}-1_{01}\right)$, both in terms of spatial distribution and the line profiles in the high-velocity range (see Fig. 2b of Lefloch et al. 2012), provides us with an estimate of the size of the water line emission, $\approx 10^{\prime \prime}$, consistent with the PACS determination. 
G. Busquet et al.: The CHESS survey of the L1157-B1 bow-shock: high and low excitation water vapor

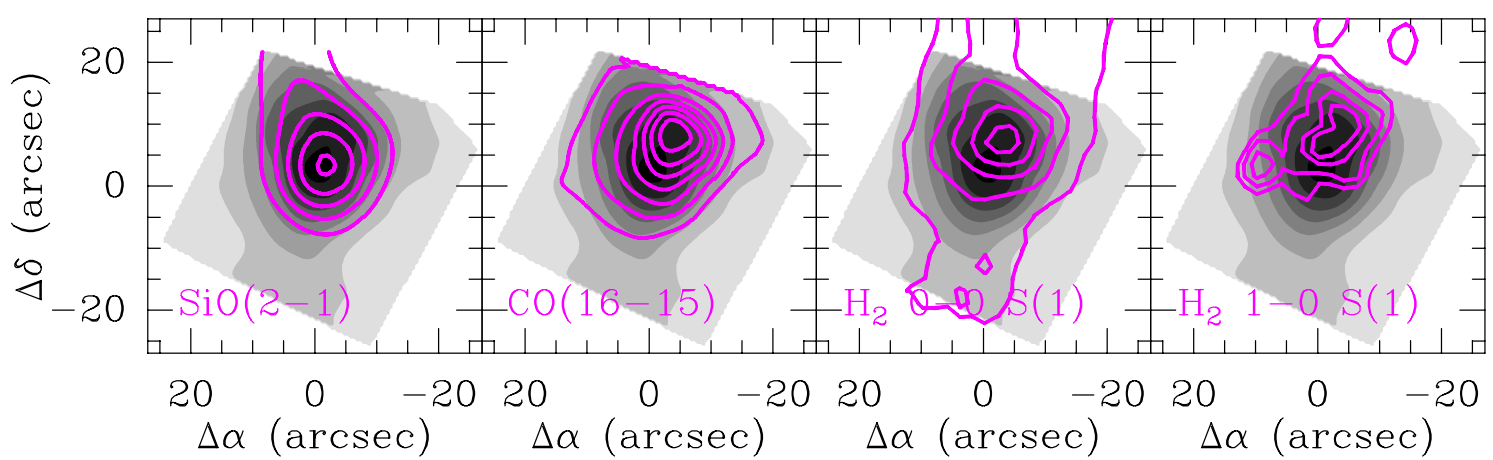

Fig. 3. Contour maps of the $\mathrm{SiO}(2-1)$ high-velocity $\left(v<-8 \mathrm{~km} \mathrm{~s}^{-1}\right)$ emission from Gueth et al. (1998) convolved to the 12 '.7 PACS resolution, the $\mathrm{CO}(16-15)$ line observed with PACS (Benedettini et al. 2012), $\mathrm{H}_{2} 0-0 \mathrm{~S}(1)$ from Nisini et al. (2010b), and $\mathrm{H}_{2} 1-0 \mathrm{~S}(1)$ from Caratti o Garatti et al. (2006), overlaid on the $\mathrm{H}_{2} \mathrm{O}\left(2_{12}-1_{01}\right)$ map at $179 \mu \mathrm{m}$ (grey scale). In each panel the transition in contours is indicated.

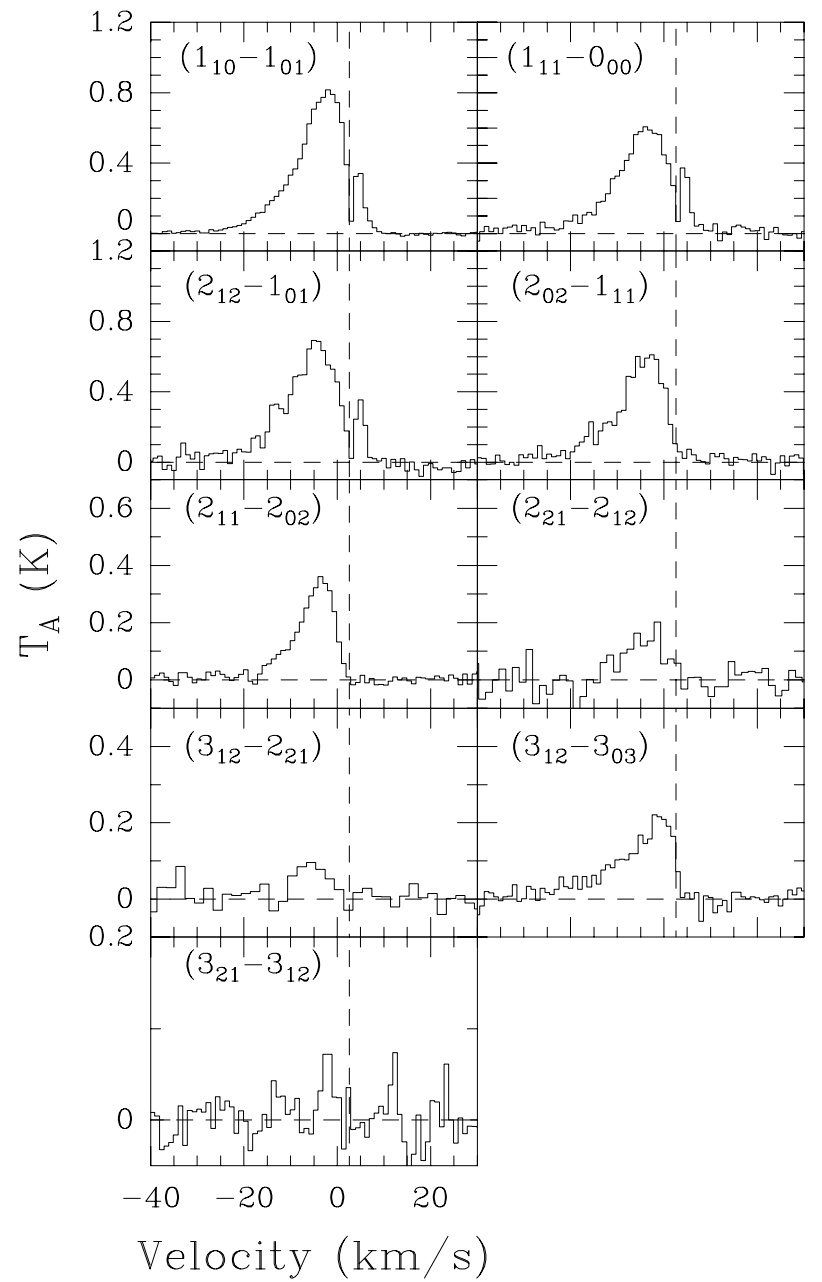

Fig. 4. HIFI $\mathrm{H}_{2} \mathrm{O}$ spectra of L1157-B1 smoothed to a velocity resolution of $1 \mathrm{~km} \mathrm{~s}^{-1}$. The $\mathrm{H}_{2} \mathrm{O}$ transition is indicated in each panel. The vertical dashed line marks the ambient LSR velocity $v_{\text {LSR }} \sim 2.6 \mathrm{~km} \mathrm{~s}^{-1}$ from $\mathrm{C}^{18} \mathrm{O}$ emission (Bachiller \& Pérez Gutiérrez 1997).

\subsection{Line profiles}

Figure 4 shows a montage of the water line spectra observed with HIFI smoothed to a velocity resolution of $1 \mathrm{~km} \mathrm{~s}^{-1}$. Water line profiles are rather broad, with a $F W H M \sim 10 \mathrm{~km} \mathrm{~s}^{-1}$. The bulk of the emission in all transitions is clearly blueshifted with respect to the cloud systemic velocity $v_{\mathrm{LSR}}=+2.6 \mathrm{~km} \mathrm{~s}^{-1}$ (Bachiller \& Pérez Gutiérrez 1997). For lines with a S/N high enough, e.g., $3_{12}-3_{03}, 3 \sigma$ emission is detected at velocities up to $-30 \mathrm{~km} \mathrm{~s}^{-1}$.

The coexistence of multiple excitation components in L1157-B1 has been studied recently by Lefloch et al. (2012), who showed, based on the spectral slope, that the CO line emission arises from three different emitting regions. These components were tentatively identified as the jet impact shock region $\left(g_{1}\right)$, the cavity walls of the L1157-B1 bow-shock $\left(g_{2}\right)$, and the cavity walls from the earlier ejection episode that produced the L1157-B2 bow-shock $\left(g_{3}\right)$. A schematic view of all these components is presented in Fig. 7. The authors showed that each component is characterized by an specific excitation temperature. We found that the profile of the $\mathrm{o}-\mathrm{H}_{2} \mathrm{O}\left(3_{12}-3_{03}\right)$ transition follows the same specific spectral signature observed for the CO (16-15) line profile (see Fig. 2b of Lefloch et al. 2012), which defines the $g_{1}$ component.

Despite the different beam sizes of the HIFI lines, Fig. 5 shows a good match between the profiles of the water lines $2_{11}-2_{02}$ at $752 \mathrm{GHz}$ and $2_{02}-1_{11}$ at $988 \mathrm{GHz}$, and between $1_{11}-0_{00}$ at $1113 \mathrm{GHz}$ and $2_{12}-1_{01}$ at $1669 \mathrm{GHz}$, respectively. This defines two groups of water lines, each of them following a specific pattern suggesting that the lines within each group arise from the same region. Whereas the lines at $1669 \mathrm{GHz}$ and $1113 \mathrm{GHz}$ both peak at $-5 \mathrm{~km} \mathrm{~s}^{-1}$, the lines at $752 \mathrm{GHz}$ and $988 \mathrm{GHz}$ peak at $-3 \mathrm{~km} \mathrm{~s}^{-1}$.

A narrow dip $\left(\Delta v=1.4 \mathrm{~km} \mathrm{~s}^{-1}\right)$ is observed at the systemic cloud velocity $v_{\text {LSR }}=2.6 \mathrm{~km} \mathrm{~s}^{-1}$ in the spectra of the three transitions that connect with the ground state level resulting in a double-peak profile (see Fig. 4 and Sect. 4.3 for further details).

Weak redshifted emission is detected in these transitions only, up to velocities of $+10 \mathrm{~km} \mathrm{~s}^{-1}$. Bjerkeli et al. (2013) showed that this weak redshifted emission is extended all over the southern outflow lobe. It is worth noting that this redshifted emission is also detected in the low- $J$ HCN and $\mathrm{HCO}^{+}$lines (Bachiller et al. 2001; Benedettini et al. 2007). The lack of emission in other tracers such as $\mathrm{CS}, \mathrm{CH}_{3} \mathrm{OH}, \mathrm{H}_{2} \mathrm{CO}$, or $\mathrm{SiO}$ suggests that this component has different excitation conditions from the main, blueshifted outflow component. The redshifted emission most likely arises from material located on the rear side of the cavity.

\subsection{Outflow emission}

Figure 6 (top panel) shows that the profile of the $\mathrm{o}-\mathrm{H}_{2} \mathrm{O}$ $\left(1_{10}-1_{01}\right)$ line at $556.9 \mathrm{GHz}$ presents a significant excess of emission at low velocities compared with the two other lines connecting the ground state level (the $2_{12}-1_{01}$ at $1669 \mathrm{GHz}$ and 


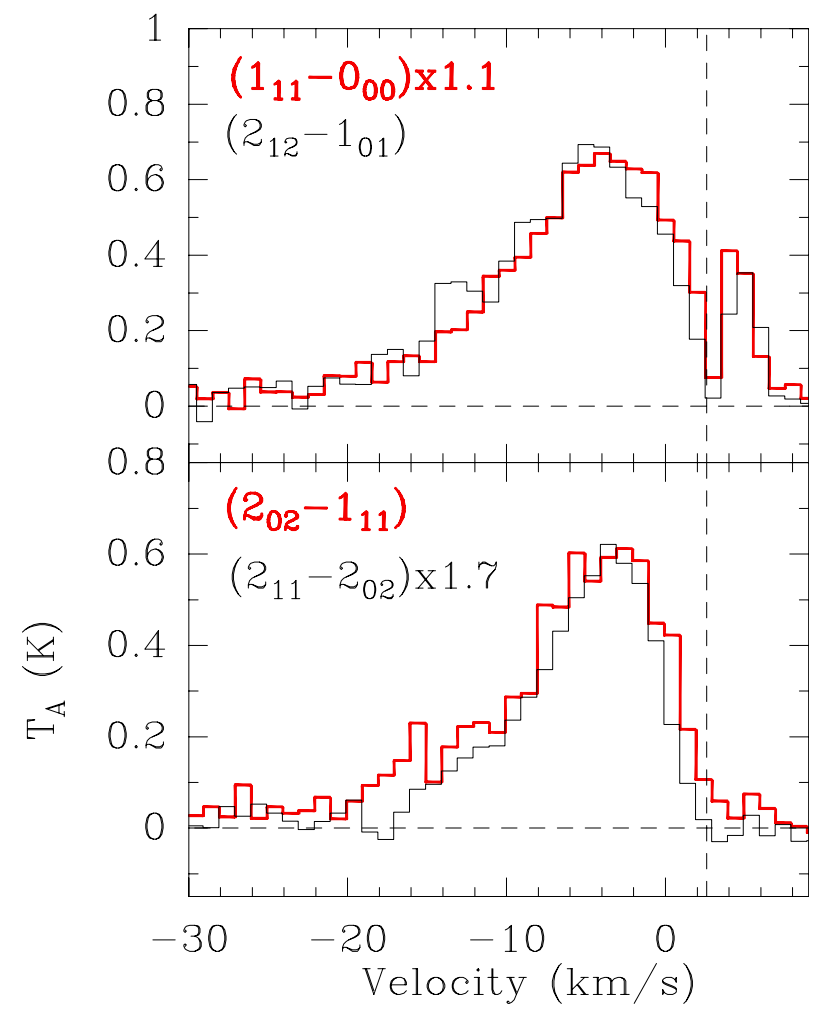

Fig. 5. Comparison of HIFI $\mathrm{H}_{2} \mathrm{O}\left(2_{12}-1_{01}\right)$ and $\left(1_{11}-0_{00}\right)$ lines (top) at $1669 \mathrm{GHz}$ and $1113 \mathrm{GHz}$, respectively, and between the $\mathrm{H}_{2} \mathrm{O}\left(2_{02}-1_{11}\right)$ at $988 \mathrm{GHz}$ and the $\left(2_{11}-2_{02}\right)$ at $752 \mathrm{GHz}$ lines (bottom). The $\mathrm{H}_{2} \mathrm{O}$ transitions are labeled in each panel. The vertical dashed line marks the ambient LSR velocity $v_{\mathrm{LSR}} \sim 2.6 \mathrm{~km} \mathrm{~s}^{-1}$ (Bachiller \& Pérez Gutiérrez 1997).

the $1_{11}-0_{00}$ at $1113 \mathrm{GHz}$ transitions) that display similar line profiles (see Fig. 5). This is surprising as the lines at $1113 \mathrm{GHz}$ and $1669 \mathrm{GHz}$ are sensitive to somewhat different excitation conditions.

We subtracted the profile of the $\mathrm{p}-\mathrm{H}_{2} \mathrm{O}\left(1_{11}-0_{00}\right)$ line from the o- $\mathrm{H}_{2} \mathrm{O}\left(1_{10}-1_{01}\right)$ line and show its residual emission in Fig. 6 (thick spectrum in the top panel). This residual emission has a peak intensity of $T_{\mathrm{mb}} \simeq 0.38 \mathrm{~K}$ and it peaks at $v=0 \mathrm{~km} \mathrm{~s}^{-1}$. This component spans a relatively narrow range of velocities, from $\sim 2.6 \mathrm{~km} \mathrm{~s}^{-1}$ up to $-8 \mathrm{~km} \mathrm{~s}^{-1}$, and its integrated intensity, in $T_{\mathrm{mb}}$ scale, is $2.3 \mathrm{~K} \mathrm{~km} \mathrm{~s}^{-1}$.

As can be seen in Fig. 1, the HIFI beam at $1113 \mathrm{GHz}$ and $1669 \mathrm{GHz}$ collects emission from a region at the apex of the bow-shock, with a typical size of $\approx 10^{\prime \prime}$ (see also Lefloch et al. 2012) whereas the $556.9 \mathrm{GHz}$ also collects emission associated with the B1 cavity walls and the entrained gas, downstream and eastward of the B1 cavity, associated with the B2 ejection. An additional clue on the origin of the extended component is obtained by comparing the $556.9 \mathrm{GHz}$ line profiles of the extended component and the older outflow cavity L1157-B2 (see also Fig. 1) observed by Vasta et al. (2012). As can be seen in Fig. 6 (bottom right panel), both profiles show an excellent match at blueshifted velocities, suggesting a common origin. Interestingly, an excellent match was observed in the CO $J=$ 3-2 profiles of the $g_{3}$ component and the L1157-B2 shock by Lefloch et al. (2012). Comparison of the line profile of the extended $\mathrm{H}_{2} \mathrm{O}$ component with the o- $\mathrm{NH}_{3}\left(1_{0}-0_{0}\right)$ and $\mathrm{CH}_{3} \mathrm{OH}$ $\left(6_{16}-5_{05}\right)$ lines (Codella et al. 2010), observed at a similar angular resolution with HIFI $\left(\sim 38^{\prime \prime}\right)$, reveals a very good agreement, supporting the hypothesis that they have a common origin and

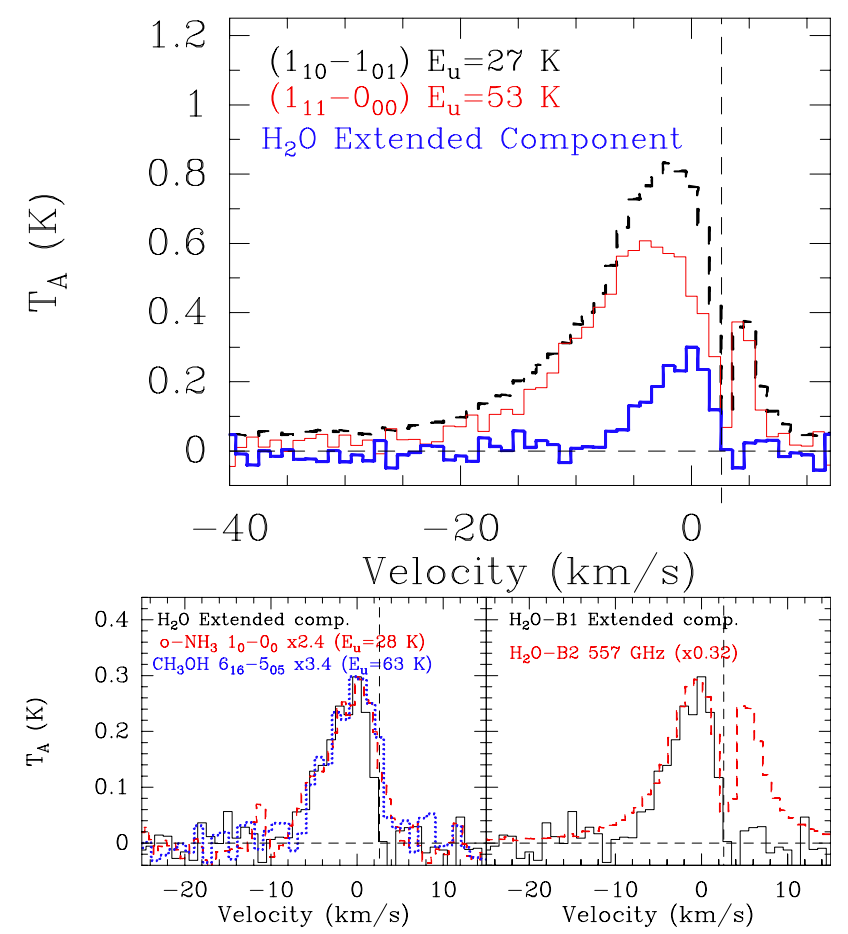

Fig. 6. Top: comparison of HIFI spectra for $\mathrm{H}_{2} \mathrm{O}$ transitions connecting with the ground state: $\mathrm{o}-\mathrm{H}_{2} \mathrm{O}\left(1_{10}-1_{01}\right)$ shown by the dashed black line and $\mathrm{p}-\mathrm{H}_{2} \mathrm{O}\left(1_{11}-0_{00}\right)$ shown by the thin solid red line. The spectrum shown in blue (solid thick line) is the residual emission after subtracting the emission of $\mathrm{p}-\mathrm{H}_{2} \mathrm{O}\left(1_{11}-0_{00}\right)$ line from the $\mathrm{o}-\mathrm{H}_{2} \mathrm{O}\left(1_{10}-1_{01}\right)$, referred to as the extended component. Bottom: comparison of the extended component seen in the $\mathrm{H}_{2} \mathrm{O}\left(1_{10}-1_{01}\right)$ line (solid black line) with the o- $\mathrm{NH}_{3}\left(1_{0}-0_{0}\right)$ (dashed red line) and the $\mathrm{CH}_{3} \mathrm{OH}\left(6_{16}-5_{05}\right)$ (dotted blue line) spectra obtained from Codella et al. (2010) (left panel). Comparison of the $\mathrm{o}-\mathrm{H}_{2} \mathrm{O}\left(1_{10}-1_{01}\right)$ line at $556.9 \mathrm{GHz}$ observed in $\mathrm{B} 1$ (extended component; solid black line) and in L1157-B2 (dashed red line) from Vasta et al. (2012). In all panels the vertical dashed line marks the ambient LSR velocity $v_{\mathrm{LSR}} \sim 2.6 \mathrm{~km} \mathrm{~s}^{-1}$ (Bachiller \& Pérez Gutiérrez 1997).

all trace the same gas. We propose that the broad HIFI beam at $556.9 \mathrm{GHz}$ is actually tracing an extended component, of low excitation, to which the beam of HIFI is less sensitive at the frequency of the $\mathrm{H}_{2} \mathrm{O}$ lines at $1113 \mathrm{GHz}(1669 \mathrm{GHz})$, as its size decreases from $38^{\prime \prime}$ to 19 !' 1 (12'.7). This component could represent the counterpart at $556.9 \mathrm{GHz}$ of the plateau observed by PACS, south of B1. Lack of angular resolution prevents us from being more specific about the origin of the extended component, and a comparison with the $556.9 \mathrm{GHz}$ map presented by Bjerkeli et al. (2013) would help to support our interpretation.

The $\mathrm{H}_{2} \mathrm{O}$ emission from the outflow has been recently analyzed by Bjerkeli et al. (2013), who presented a detailed study of the physical properties (molecular mass, dynamical time-scale, momentum, kinetic energy, etc.) in the outflow using $\mathrm{CO}$ and $\mathrm{H}_{2} \mathrm{O}$ lines. In what follows, we will concentrate on the physical conditions in the bow-shock, where the bulk of $\mathrm{H}_{2} \mathrm{O}$ emission originates.

\section{Analysis and discussion}

We have determined the physical conditions of the B1 shock region by modeling the water line emission using a radiative transfer code in the large velocity gradient (LVG) approximation. It is worth noting that the $\mathrm{B} 1$ shock position is about 1.5 far away 


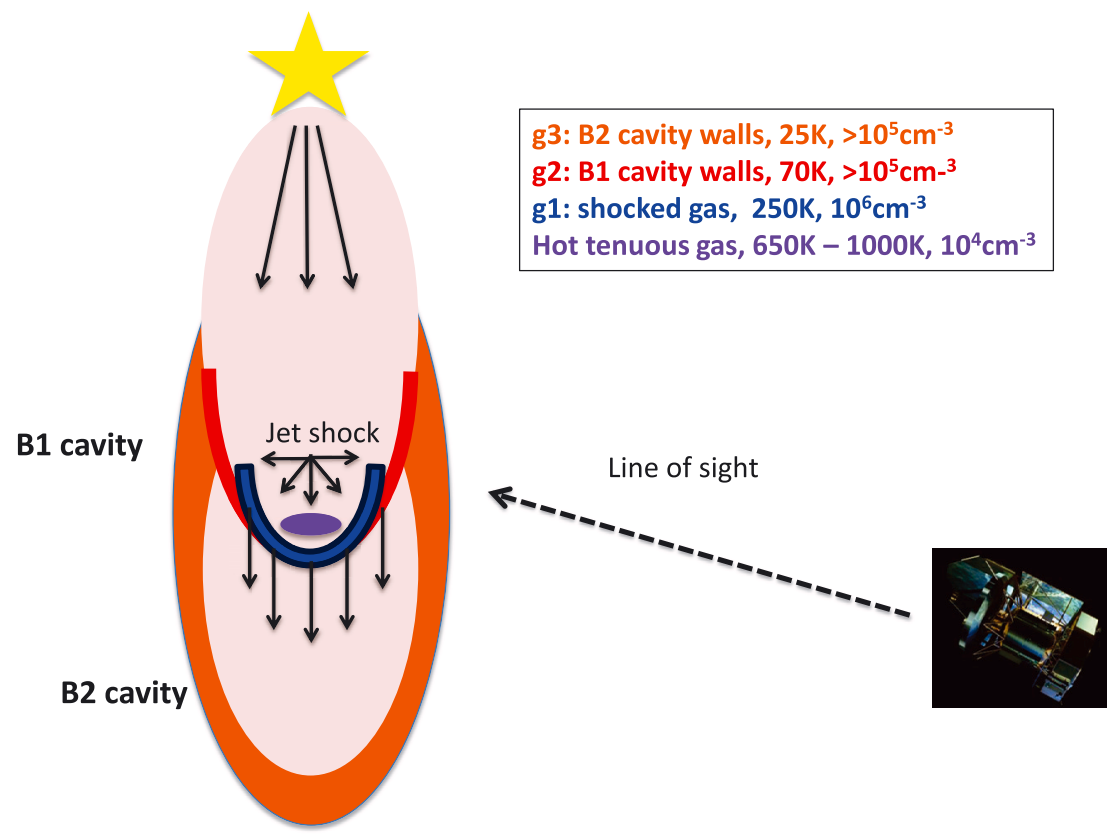

Fig. 7. L1157 blue-lobe outflow system. The B1 and B2 outflow cavities are indicated in red and orange, respectively. The two shock components identified through $\mathrm{H}_{2} \mathrm{O}$ lines are displayed in blue (warm shocked gas) and in light violet for the hot tenuous gas. In the top right corner of the image we show the physical conditions of each component. The observer, represented by the Herschel satellite, is indicated on the right side of the image.

from the protostar L1157-mm; the continuum emission detected in the submm/far-IR range is faint enough that infrared radiative pumping of the $\mathrm{H}_{2} \mathrm{O}$ lines can be neglected.

We first present our approach to the modeling of the L1157-B1 emission (Sect. 4.1), we then discuss the best-fit solution to the emission from the B1 shock (Sect. 4.2), and we show its consistency with the previous works on $\mathrm{CO}$ and $\mathrm{H}_{2}$. We assess the influence of various parameters of the modeling, in particular the actual value of the ortho to para $\mathrm{H}_{2}$ ratio in the shocked gas. Finally, we study the origin of the water absorbing layer in the cloud (Sect. 4.3), and report on the water abundance and far-IR cooling (Sect. 4.4). For the sake of clarity, in Fig. 7 we display the physical structure emerging from our $\mathrm{H}_{2} \mathrm{O}$ line study that summarizes the main results to be presented in this section. Briefly, we identified five components: the cloud seen in absorption, the outflow material from the B1 $\left(g_{2}\right)$ and B2 $\left(g_{3}\right)$ cavities, the jet impact shock region $\left(g_{1}\right)$, and a compact hot gas component.

\subsection{Modeling}

Previous studies at millimeter and infrared wavelengths (Benedettini et al. 2007; Codella et al. 2009; Takami et al. 2011; and more recently Benedettini et al. 2013) reveal a complex density, temperature, and velocity structure, with several emission knots of shocked gas in various tracers. In such a complex environment, a comprehensive modeling of the water line emission from the bow-shock, notoriously a very difficult task, is just too difficult to handle if one considers the angular resolution of the data (at best comparable to the size of the region), the onedimensional nature of the source geometrical modeling, and the radiative transfer code used. Our goal here is to identify the main shock components responsible for the $\mathrm{H}_{2} \mathrm{O}$ emission detected, and, within the uncertainties inherent to the calibration and geometry adopted, the physical conditions of these components.

The PACS maps (Fig. 2) show that the $\mathrm{H}_{2} \mathrm{O}$ emission does not peak at the nominal position of $\mathrm{B} 1$, where the HIFI beam is centered; the larger HIFI beams encompass the emission peak while for the smaller HIFI beams the peak is partially covered (see Fig. 1). It is all the more important to carry out LVG calculations using water fluxes measured over the same source solid angle; we therefore convolved all the PACS maps to a common angular resolution of 12 '.7 to measure the flux towards the nominal position of B1. For the HIFI lines, we convolved the o- $\mathrm{H}_{2} \mathrm{O}\left(2_{12}-1_{01}\right)$ PACS map at the resolution of the different HIFI beams. Assuming that all HIFI lines have the same spatial distribution as the o- $\mathrm{H}_{2} \mathrm{O}\left(2_{12}-1_{01}\right)$ line we can then derive the HIFI beam filling factor. As a test, we compared the beam filling factors obtained from PACS maps of the 108, 138, and $125 \mu \mathrm{m}$ lines. In practice, we obtain very similar correcting factors, which made us feel confident about the robustness of the procedure and the results obtained. The fluxes of all the $\mathrm{H}_{2} \mathrm{O}$ lines estimated in a beam of $12^{\prime \prime} .7$ are listed in Table 3.

We investigated the excitation conditions of the $\mathrm{H}_{2} \mathrm{O}$ line emission using a radiative transfer code in the LVG approximation (Ceccarelli et al. 2003) and adopting a plane parallel geometry. The molecular data were taken from the BASECOL ${ }^{4}$ database (Dubernet et al. 2006, 2013) and we used the new collisional rate coefficients with $\mathrm{H}_{2}$ (Dubernet et al. 2009; Daniel et al. 2010, 2011). The linewidth (FWHM of the line profile) was set to a fixed value of $10 \mathrm{~km} \mathrm{~s}^{-1}$. The model includes the effects of the beam filling factor, and it computes the reduced chi-square $\chi_{\mathrm{r}}^{2}$ for each column density minimizing with respect to the source size, kinetic temperature, and density. We adopted an uncertainty in the integrated intensities of $30 \%$ for all lines

4 http://basecol.obspm.fr 
Table 3. Observed and predicted water line fluxes for an $\mathrm{OPR} \mathrm{H}_{2} \mathrm{O}=3$.

\begin{tabular}{|c|c|c|c|c|c|c|c|}
\hline \multirow[b]{2}{*}{ Line } & \multirow[b]{2}{*}{$\begin{array}{l}\text { Freq. } \\
(\mathrm{GHz})\end{array}$} & \multirow[b]{2}{*}{$\begin{array}{c}\lambda \\
(\mu \mathrm{m})\end{array}$} & \multirow[b]{2}{*}{$\begin{array}{c}E_{\mathrm{u}} / k_{\mathrm{B}} \\
(\mathrm{K})\end{array}$} & \multirow[b]{2}{*}{$\begin{array}{c}\text { Flux[12.7"1 }]^{\mathrm{a}} \\
\left.(\mathrm{K} \mathrm{km} \mathrm{s})^{-1}\right)\end{array}$} & \multicolumn{3}{|c|}{ LVG predictions } \\
\hline & & & & & $\begin{array}{l}\text { Comp. 1-warm } \\
\left(\mathrm{K} \mathrm{km} \mathrm{s}^{-1}\right)\end{array}$ & $\begin{array}{c}\text { Comp. 2-hot }{ }^{\mathrm{c}} \\
\left(\mathrm{K} \mathrm{km} \mathrm{s}^{-1}\right)\end{array}$ & $\begin{array}{c}\text { Total } \\
\left(\mathrm{K} \mathrm{km} \mathrm{s}^{-1}\right) \\
\end{array}$ \\
\hline \multicolumn{8}{|l|}{$\mathrm{o}-\mathrm{H}_{2} \mathrm{O}$} \\
\hline $1_{10}-1_{01}$ & 556.936 & 538.66 & 26.7 & $35.0(13.0)^{\mathrm{d}}$ & 39.0 & 8.3 & 47.3 \\
\hline $3_{12}-3_{03}$ & 1097.365 & 273.38 & 215.1 & $6.0(2.0)$ & 0.3 & 1.0 & 1.3 \\
\hline $3_{12}-2_{21}$ & 1153.127 & 260.17 & 215.1 & $1.9(0.9)$ & 0.05 & 1.4 & 1.5 \\
\hline $3_{21}-3_{12}$ & 1162.912 & 257.98 & 271.0 & $0.8(0.4)$ & 0.02 & 0.9 & 0.9 \\
\hline $22_{21}-2_{12}$ & 1661.008 & 180.49 & 159.8 & $3.0(1.0)$ & 0.2 & 1.6 & 1.8 \\
\hline $2_{12}-1_{01}$ & 1669.905 & 179.52 & 80.1 & $14.0(4.0)$ & 9.2 & 2.7 & 11.9 \\
\hline $3_{03}-2_{12}$ & 1716.770 & 174.62 & 162.5 & $6.0(2.0)$ & 1.0 & 3.4 & 4.4 \\
\hline $4_{14}-3_{03}$ & 2640.474 & 113.54 & 289.3 & $0.6(0.3)$ & 0.06 & 0.4 & 0.5 \\
\hline $22_{12}-1_{10}$ & 2773.977 & 108.10 & 159.9 & $0.6(0.2)$ & 0.4 & 0.6 & 1.0 \\
\hline \multicolumn{8}{|l|}{ p- $\mathrm{H}_{2} \mathrm{O}$} \\
\hline $2_{11}-2_{02}$ & 752.033 & 398.92 & 136.9 & $11.4(3.3)$ & 1.3 & 6.2 & 7.5 \\
\hline $2_{02}-1_{11}$ & 987.927 & 303.67 & 100.8 & $19.0(6.0)$ & 2.9 & 8.4 & 11.3 \\
\hline $1_{11}-0_{00}$ & 1113.343 & 269.47 & 53.4 & $21.0(6.0)$ & 12.8 & 6.3 & 19.1 \\
\hline $3_{13}-2_{02}$ & 2164.132 & 138.54 & 207.7 & $1.1(0.3)$ & 0.1 & 1.0 & 1.1 \\
\hline
\end{tabular}

Notes. Uncertainties are indicated in parentheses. ${ }^{(a)}$ Corrected fluxes obtained for a common angular resolution of 12:.7. ${ }^{(b)}$ LVG predicted fluxes for the best-fit model of the warm component $\left(T_{\text {kin }}=250 \mathrm{~K}, n\left(\mathrm{H}_{2}\right)=10^{6} \mathrm{~cm}^{-3}, N\left(\mathrm{o}-\mathrm{H}_{2} \mathrm{O}\right)=2 \times 10^{14} \mathrm{~cm}^{-2}, N\left(\mathrm{p}-\mathrm{H}_{2} \mathrm{O}\right)=7.0 \times 10^{13} \mathrm{~cm}{ }^{-2}\right.$, and a source size of $10^{\prime \prime}$ ) and using an OPR $\mathrm{H}_{2}$ of 0.5 (see Sects. 4.2.1. and 4.2.2). ${ }^{(c)} \mathrm{LVG}$ predicted fluxes for the best-fit model of the hot component $\left(T_{\text {kin }}=1000 \mathrm{~K}, n\left(\mathrm{H}_{2}\right)=2 \times 10^{4} \mathrm{~cm}^{-3}, N\left(\mathrm{o}-\mathrm{H}_{2} \mathrm{O}\right)=7 \times 10^{16} \mathrm{~cm}^{-2}, N\left(\mathrm{p}-\mathrm{H}_{2} \mathrm{O}\right)=2.1 \times 10^{16} \mathrm{~cm}^{-2}\right.$, and a source size of 2'.5) and using an OPR $\mathrm{H}_{2}$ of 3 (see Sects. 4.2.1. and 4.2.2). ${ }^{(d)}$ Flux after removing the contribution of the extended component detected in the 556.9 GHz line (see Sect. 3.3).

except the HIFI lines with high $E_{\mathrm{u}}$ and the PACS lines lying at the edges of the band, for which the adopted uncertainty is $50 \%$.

\subsection{Shock emission: best-fit model}

\subsubsection{A two-temperature model}

The present LVG calculations were carried out for an ortho-topara ratio (OPR) of 0.5 in the $\mathrm{H}_{2}$ gas, which is close to the value estimated by Nisini et al. (2010b) for gas in the same range of excitation conditions from modeling the $\mathrm{H}_{2}$ emission, and an OPR of the $\mathrm{H}_{2} \mathrm{O}$ gas equal to 3 . We also adopted a size of $10^{\prime \prime}$, as estimated from the PACS maps, consistent with our previous findings (Benedettini et al. 2012) and with the Spitzer image of L1157-B1 observed in the $\mathrm{H}_{2}$ lines (Nisini et al. 2010b) and in the IRAC bands (Takami et al. 2011).

To account for the line fluxes of the three transitions connected to the ground state (ortho and para) and assuming a source size of $10^{\prime \prime}$, the acceptable range of physical condition is $T_{\text {kin }} \sim 200-300 \mathrm{~K}, n\left(\mathrm{H}_{2}\right)=(1-6) \times 10^{6} \mathrm{~cm}^{-3}$, and $N\left(\mathrm{o}-\mathrm{H}_{2} \mathrm{O}\right)=$ $(0.8-2) \times 10^{14}$. The best-fit model yields a warm gas component at $250 \mathrm{~K}, n\left(\mathrm{H}_{2}\right)=1 \times 10^{6} \mathrm{~cm}^{-3}, N\left(\mathrm{o}-\mathrm{H}_{2} \mathrm{O}\right)=2 \times 10^{14} \mathrm{~cm}^{-2}$. These physical conditions are absolutely unable to account for the flux of lines at higher upper energy levels (see Fig. 8, top panel).

A second gas component, at a much higher temperature and lower density, is needed to reproduce the flux detected in the higher $E_{\mathrm{u}}$ transitions. Solutions with $T_{\text {kin }} \simeq 650 \mathrm{~K}, N\left(\mathrm{o}-\mathrm{H}_{2} \mathrm{O}\right)=$ $1 \times 10^{17} \mathrm{~cm}^{-2}, n\left(\mathrm{H}_{2}\right)=8 \times 10^{3} \mathrm{~cm}^{-3}$, and a source size of $4^{\prime \prime}$, are possible, in principle, under the assumption that the $\mathrm{OPR}-\mathrm{H}_{2}$ remains unchanged, equal to 0.5 . Observational constraints on $\mathrm{H}_{2}$ suggest a higher value of $\mathrm{OPR}-\mathrm{H}_{2}$, typically $\simeq 3$, at high temperatures (see below). Assuming a typical OPR- $\mathrm{H}_{2}$ of 3 for the second, hot gas component, our modeling favor higher-temperature solutions, with $T_{\text {kin }} \simeq 1000 \mathrm{~K}, N\left(\mathrm{o}-\mathrm{H}_{2} \mathrm{O}\right)=$ $7 \times 10^{16} \mathrm{~cm}^{-2}, n\left(\mathrm{H}_{2}\right)=2 \times 10^{4} \mathrm{~cm}^{-3}$, and a size of $2^{\prime \prime} .5$ as a best-fit model. We found, however, a range of possible solutions, with $T_{\text {kin }} \sim 900-1400 \mathrm{~K}, N\left(\mathrm{o}-\mathrm{H}_{2} \mathrm{O}\right)=(3-7) \times 10^{16} \mathrm{~cm}^{-2}$, $n\left(\mathrm{H}_{2}\right)=(0.8-2) \times 10^{4} \mathrm{~cm}^{-3}$, and a size of $2^{\prime \prime}-5^{\prime \prime}$.

Therefore, in addition to the warm dense $g_{1}$ gas, a small region of hot, low density gas is contributing to the water emission detected by Herschel.

To evaluate the quality of our best-fit model, we have computed the ratio of the measured water line fluxes to those predicted by our model as a function of the upper energy level of the transition. As can be seen in Fig. 8 (top panel), the overall agreement between the measured fluxes and the observations is satisfying; a $\chi_{\mathrm{r}}^{2}$ minimization of our two-temperature model yields $\chi_{\mathrm{r}}^{2}=1.0$. The water line fluxes resulting from the LVG modeling are reported in Table 3 and the range of physical conditions of the warm $\left(g_{1}\right)$ and hot shock gas components are summarized in Table 4. The large number of lines detected at high $\mathrm{S} / \mathrm{N}$ together with the availability of a wealth of complementary data allow us to constrain the water excitations conditions with unprecedented precision. It is important, however, to note that source sizes have been imposed and this two-component model is a simplification of the complex structure of the bow-shock, in which a wide and continuous range of temperatures and densities are most likely present.

Finally, we evaluated the influence of the OPR- $\mathrm{H}_{2} \mathrm{O}$ on the results. Only models with an OPR- $\mathrm{H}_{2} \mathrm{O}$ of 3 (the statistical equilibrium value) can match the observed line fluxes, while a value of 1 always yields solutions with a $\chi_{\mathrm{r}}^{2}>2$.

\subsubsection{Influence of the ortho-to-para $\mathrm{H}_{2}$ ratio}

In their study of the emission of the pure rotational lines of $\mathrm{H}_{2}$ with Spitzer, Nisini et al. (2010b) found evidence for two gas components at $\approx 300 \mathrm{~K}$ and $1400 \mathrm{~K}$, respectively. They modeled the OPR $-\mathrm{H}_{2}$ as varying continuously from a value of $\approx 0.6$ in gas at $300 \mathrm{~K}$ to its value at LTE $(=3)$ in gas at $1400 \mathrm{~K}$. Therefore, 
Table 4. Physical conditions of the shock components accounting for the water line emission in L1157-B1.

\begin{tabular}{lccccccccc}
\hline \hline Comp. & $\begin{array}{c}T_{\text {kin }} \\
(\mathrm{K})\end{array}$ & $\begin{array}{c}n\left(\mathrm{H}_{2}\right) \\
\left(\mathrm{cm}^{-3}\right)\end{array}$ & $\begin{array}{c}N\left(\mathrm{H}_{2} \mathrm{O}\right) \\
\left(\mathrm{cm}^{-2}\right)\end{array}$ & $\begin{array}{c}N\left(\mathrm{H}_{2}\right) \\
\left(\mathrm{cm}^{-2}\right)\end{array}$ & $X\left(\mathrm{H}_{2} \mathrm{O}\right)$ & $\begin{array}{c}\text { Size } \\
\left({ }^{\prime}\right)\end{array}$ & $\begin{array}{c}L\left(\mathrm{H}_{2} \mathrm{O}\right) \\
\left(L_{\odot}\right)\end{array}$ & $\begin{array}{c}L(\mathrm{CO}) \\
\left(L_{\odot}\right)\end{array}$ & $\begin{array}{c}{\left[\mathrm{H}_{2} \mathrm{O}\right] /[\mathrm{CO}]} \\
\end{array}$ \\
\hline Warm & $250-300$ & $(1-3) \times 10^{6}$ & $(1.2-2.7) \times 10^{14}$ & $1.2 \times 10^{20}$ & $(0.7-2.0) \times 10^{-6}$ & 10 & 0.002 & 0.004 & 0.03 \\
Hot & $900-1400$ & $(0.8-2) \times 10^{4}$ & $(4.0-9.1) \times 10^{16}$ & $3.3 \times 10^{20}$ & $(1.2-3.6) \times 10^{-4}$ & $2-5$ & 0.03 & 0.01 & 1 \\
\hline
\end{tabular}
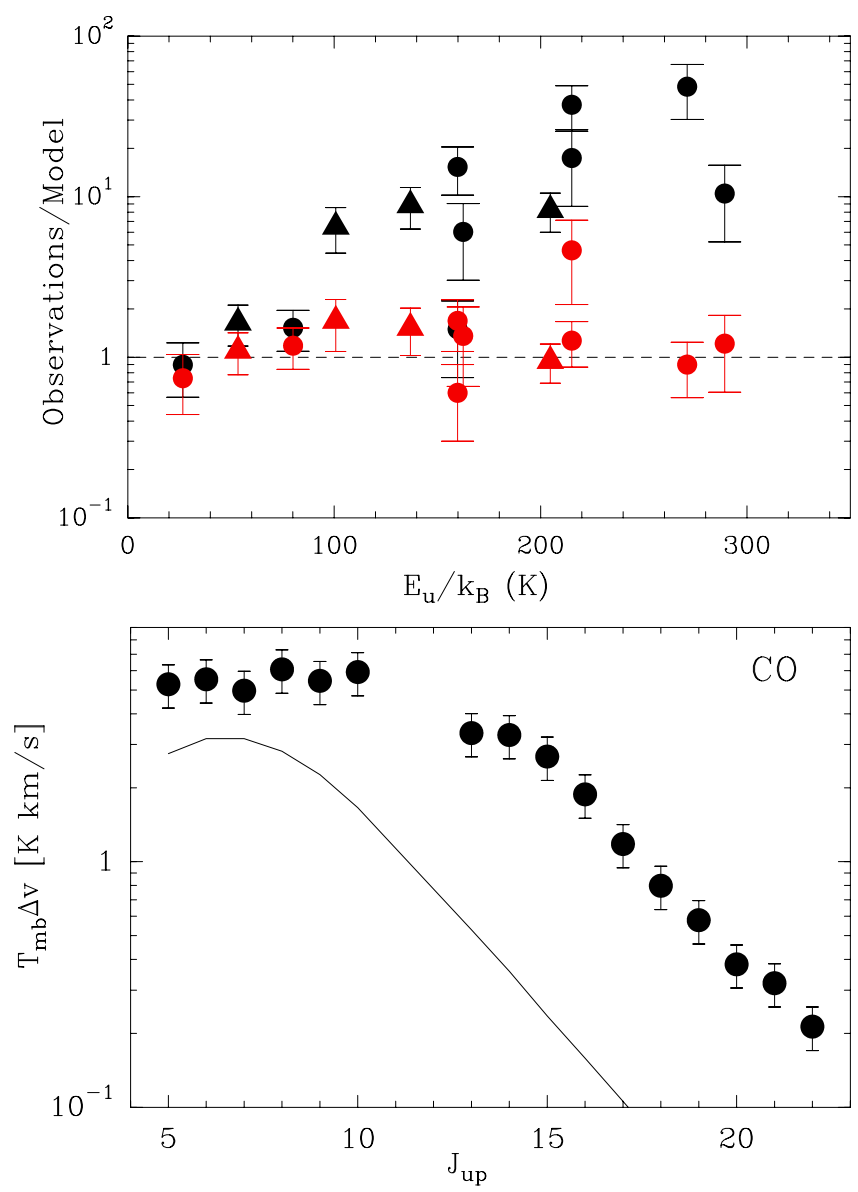

Fig. 8. Top: ratio between the measured integrated intensities and the LVG model predictions. Filled circles/triangles depict $\mathrm{o}-\mathrm{H}_{2} \mathrm{O}$ and $\mathrm{p}-\mathrm{H}_{2} \mathrm{O}$ lines, respectively. In black, we display the results assuming one single temperature component $\left(T_{\text {kin }}=250 \mathrm{~K}, n\left(\mathrm{H}_{2}\right)=10^{6} \mathrm{~cm}^{-3}\right.$, $N\left(\mathrm{o}-\mathrm{H}_{2} \mathrm{O}\right)=2 \times 10^{14} \mathrm{~cm}^{-2}$, size $\left.=10^{\prime \prime}\right)$; in red, the final solution when adding the contribution of the second temperature $\left(T_{\text {kin }}=1000 \mathrm{~K}\right.$, $n\left(\mathrm{H}_{2}\right)=2 \times 10^{4} \mathrm{~cm}^{-3}, N\left(\mathrm{o}-\mathrm{H}_{2} \mathrm{O}\right)=7 \times 10^{16} \mathrm{~cm}^{-2}$, size $=2$ '. 5$)$. Bottom: predicted $\mathrm{CO}$ fluxes from the hot component, as a function of the rotational upper level in the HIFI and PACS range. The observed fluxes (Benedettini et al. 2012; Lefloch et al. 2012) are marked with filled circles. The choice of $[\mathrm{CO}] /\left[\mathrm{H}_{2} \mathrm{O}\right]=1$ provides an upper limit estimate of the CO integrated intensity (see Sect. 4.2.3 for more details).

we explored the range of acceptable solutions $(n, N, T)$ when considering $\mathrm{OPR}-\mathrm{H}_{2}$ as a free parameter. The best-fit solution was obtained for an OPR of 0.5 in the gas at $250 \mathrm{~K}$, hence a value similar to that found by Nisini et al. (2010b) in the gas of moderate excitation. We could not find a reasonable set of physical conditions for values of OPR- $\mathrm{H}_{2}$ higher than 1 for that component.

As noticed by Wilgenbus et al. (2000), such a low value of the OPR $-\mathrm{H}_{2}$ indicates that the gas has been recently heated up by the passage of the shock front, and not affected by an older shock episode since the timescale between shock episodes is much smaller than the timescale needed for the OPR- $\mathrm{H}_{2}$ to return to the equilibrium value. This is consistent with the youth of B1, for which the estimated dynamical age is $\sim 2000 \mathrm{yr}$ (Gueth et al. 1996) and the evolutionary age of the shock model presented by Gusdorf et al. (2008b). Low values of the OPR- $\mathrm{H}_{2}$ have been reported in other outflow shock regions (e.g., Neufeld et al. 1998, 2006; Lefloch et al. 2003; Maret et al. 2009).

As for the second, hot gas component contributing to the water line emission, higher-temperature solutions are favored when adopting an OPR- $\mathrm{H}_{2}$ of 3 , and we found satisfying solutions $\left(\chi_{\mathrm{r}}^{2}=0.8-1.2\right)$ for $T_{\mathrm{kin}} \simeq 1000 \mathrm{~K}$, and a gas column density $N\left(\mathrm{H}_{2} \mathrm{O}\right) \simeq 9 \times 10^{16} \mathrm{~cm}^{-2}$. The density and the size are less well constrained, with values of the order a few $10^{3-4} \mathrm{~cm}^{-3}$ and a few arcsec, respectively.

\subsubsection{Modeling consistency}

Since our simple model aims at reproducing only the water line fluxes and not the line profiles, one might question its consistency with respect to the spectroscopic information of the line profiles obtained with HIFI. In other words, is there any evidence for specific observational signatures of the two temperature components invoked in our modeling?

From Fig. 8 (top panel), it appears immediately that the bulk of flux of most of the lines in the HIFI and PACS range actually comes from the hot gas component at $T_{\text {kin }} \approx 1000 \mathrm{~K}$. Conversely, the lines at 556.9, 1669, and $1113 \mathrm{GHz}$ (HIFI) are very well accounted for by the warm component at $T_{\text {kin }} \approx 250 \mathrm{~K}$. This is consistent with the two groups of line profiles $(1113 / 1669 \mathrm{GHz}$ and 752/998 GHz) identified (see Fig. 5 in Sect. 3.2). The line profiles are very similar within each group, and differ markedly from one group to the other. Our model provides a simple explanation to this observational fact: we are actually probing two different regions with different excitation conditions.

Second, we have compared our PACS observations with the fluxes predicted by our two-temperature model for all the water lines falling in the range 50-200 $\mu \mathrm{m}$. As can be seen in Fig. 9, most of the lines remain below the dashed line, which draws the sensitivity limit of the observations. Our model does not predict more lines lying above the sensitivity limit than those actually detected.

The CO line observations with PACS and HIFI revealed a warm, dense gas component, thermalized at $220 \mathrm{~K}$, which Benedettini et al. (2012) and Lefloch et al. (2012) attributed to the jet impact region against the B1 cavity. Both the location and the temperature of this component agree with the properties with the warm gas component identified by Nisini et al. (2010b). However, since the bulk of emission of the CO (16-15) and $\mathrm{H}_{2} \mathrm{O} 1097 \mathrm{GHz}$ lines arises from two components of different excitation inside the $\mathrm{B} 1$ cavity, we conclude that the profile of the $\mathrm{H}_{2} \mathrm{O} 1097 \mathrm{GHz}$ line might actually not be specific to $g_{1}$ and that it indicates a more complex origin of that spectral feature, the opposite of what was claimed in a previous work (Lefloch et al. 2012). 


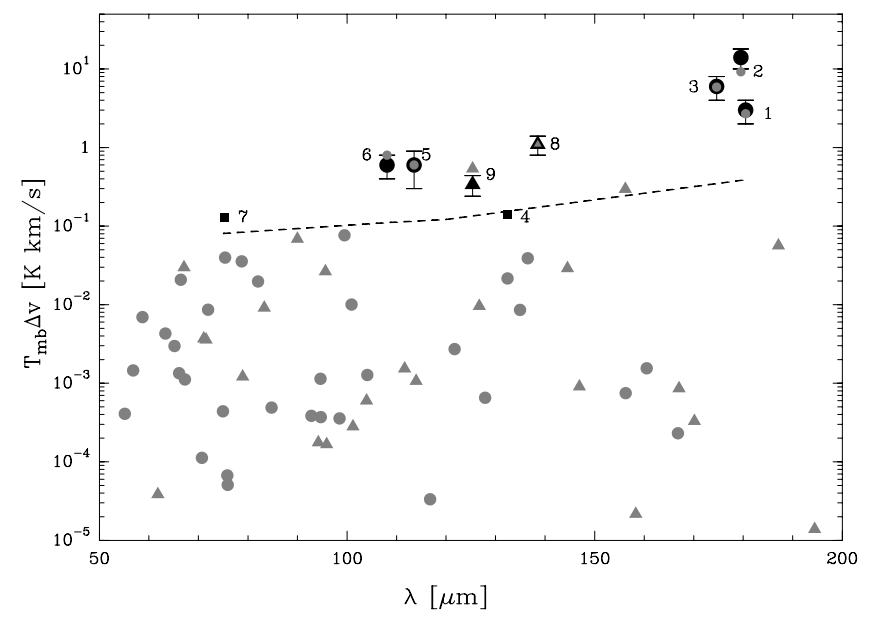

Fig. 9. Predicted $\mathrm{H}_{2} \mathrm{O}$ fluxes in the PACS range of the two-component model, shown in Fig. 8 and Table 3 , as a function of wavelength. Black/gray circles and triangles mark the observed/predicted fluxes of $\mathrm{o}-\mathrm{H}_{2} \mathrm{O}$ and $\mathrm{p}-\mathrm{H}_{2} \mathrm{O}$ lines, respectively. Squares represent upper limits of $\mathrm{H}_{2} \mathrm{O}$ lines listed in Table 2. The line_ID is also shown (see Table 2). The dashed line indicates the sensitivity limit of PACS.

One might wonder why the CO counterpart of the second component is not detected by the sensitive PACS and HIFI instruments. This is illustrated in the bottom panel in Fig. 8, which displays the predicted $\mathrm{CO}$ fluxes for the hot component $\left(T_{\mathrm{kin}} \approx\right.$ $1000 \mathrm{~K}, n\left(\mathrm{H}_{2}\right)=2 \times 10^{4} \mathrm{~cm}^{-3}$, and the assumed size of $\left.2 . ' 5\right)$, assuming an abundance ratio $[\mathrm{CO}] /\left[\mathrm{H}_{2} \mathrm{O}\right]=1$. Adopting the standard value of $10^{-4}$ for $\mathrm{CO}$ would imply a smaller value of the column density, and the flux of the hot component would be even smaller.

We point out that a similar two-component model has recently been presented by Santangelo et al. (2013) to account for the water emission towards the B2 shock position of the L1448 molecular outflow, where the physical conditions are similar to the ones obtained in L1157-B1. Confirming the presence of similar two-component structures in other shock regions would suggest that both components are most likely related to the bow-shock phenomenon itself. The nature of the relation could provide some clues into the origin of the line profiles observed.

\subsection{Cloud absorption}

In Sect. 3.2, we showed that $\mathrm{H}_{2} \mathrm{O}$ transitions connecting with the ground state level present a narrow self-absorption feature close to the ambient velocity, at $v_{\mathrm{LSR}} \sim 2.6 \mathrm{~km} \mathrm{~s}^{-1}$. Since optically thin lines, such as ${ }^{13} \mathrm{CO}$ (Lefloch et al., in prep.) and HDO (Codella et al. 2012), peak close to the ambient velocity, we propose that the absorption feature seen in the low-excited $\mathrm{H}_{2} \mathrm{O}$ lines most likely arises from an extended layer associated with the cloud envelope, as a result of ice photodesorption. This model was successfully applied by Coutens et al. (2012) to the low-mass Class 0 protostar IRAS 16293-2422, where the authors found a similar self-absorption signature in the fundamental lines of $\mathrm{HDO}$ and $\mathrm{H}_{2}^{18} \mathrm{O}$. To account for the observed line profiles, the authors added an absorbing layer in front of the IRAS 16293-2422 envelope that results from the photodesorption of icy mantles at the edge of the cloud by the far-ultraviolet (FUV) photons, as modeled by Hollenbach et al. (2009).

Interpreting the absorption feature in a similar way as due to a water-rich layer caused by ice photodesorption at the cloud surface, we can estimate its water abundance. Caratti o Garatti et al. (2006) evaluate the visual extinction towards the B1 shock through near-IR data and find that $A_{V}$ is, at most, 2 mag. Thus, adopting $A_{V}$ of 1-2 mag, assuming an incident FUV flux of $G_{0}=1$ (i.e., adopting a standard interstellar radiation field), and a typical cloud gas density of $10^{4} \mathrm{~cm}^{-3}$, Hollenbach et al. (2009) predict a water abundance of about $\sim 10^{-7}$. At the same depth and for a fixed value of $G_{0}$, lower densities would result in slightly lower values of the $\mathrm{H}_{2} \mathrm{O}$ abundance. Instead, if we consider higher values of $G_{0}$ the water abundance for $A_{V}$ of 1-2 mag will be lower as a higher incident flux modifies the depth of the freeze, moving it towards higher visual extinction, resulting in a peak deeper in the cloud for gas-phase $\mathrm{H}_{2} \mathrm{O}$. Therefore, we estimate the water abundance of the cloud absorbing layer to be $\simeq 10^{-7}$. Adopting the relation $N\left(\mathrm{H}_{2}\right)=9.4 \times 10^{20} A_{V}$ (Frerking et al. 1982) we obtained $N\left(\mathrm{H}_{2}\right)=(0.9-1.9) \times 10^{21} \mathrm{~cm}^{-2}$ for an $A_{V}$ of 1-2 mag, and hence the column density of $\mathrm{H}_{2} \mathrm{O}$ in this absorbing layer should be about $1 \times 10^{13} \mathrm{~cm}^{-2}$.

\subsection{Water abundance and line cooling}

The water abundance $X\left(\mathrm{H}_{2} \mathrm{O}\right)=\left[\mathrm{H}_{2} \mathrm{O}\right] /\left[\mathrm{H}_{2}\right]$ in the shocked gas was derived using the $\mathrm{H}_{2}$ data from Nisini et al. (2010b) obtained with Spitzer and convolved at the PACS resolution (12.7). For the $250 \mathrm{~K}$ gas component, the $\mathrm{H}_{2}$ column density was computed from an LTE analysis of the $S(0)$ to $S(2)$ rotational lines. For a source size of $10^{\prime \prime}$, the column density of $\mathrm{H}_{2}$ is $N\left(\mathrm{H}_{2}\right) \simeq 1.2 \times 10^{20} \mathrm{~cm}^{-2}$, which yields a fractional abundance of water $\sim(0.7-2) \times 10^{-6}$. This warm gas component is associated with a partly dissociative J-type shock with shock velocities and pre-shock densities, respectively, of either $v>$ $30 \mathrm{~km} \mathrm{~s}^{-1}$ and $2 \times 10^{4} \mathrm{~cm}^{-3}$, or $v>20 \mathrm{~km} \mathrm{~s}^{-1}$ and $2 \times 10^{5} \mathrm{~cm}^{-3}$ (Benedettini et al. 2012; Lefloch et al. 2012), and hence the low water abundance can be explained in terms of FUV photons produced at the shock front; these photons prevent the full conversion of free oxygen into water, and result in a decrease of the water abundance. To obtain the water abundance of the hot gas, we considered the $\mathrm{H}_{2}$ rotational lines $\mathrm{S}(5)$ up to $\mathrm{S}(7)$ and computed the $\mathrm{H}_{2}$ column density scaled for a source size of 2 ". 5 . The derived water abundance is $(1.2-3.6) \times 10^{-4}$, in agreement with the predicted values for hot shocked material (Kaufman \& Neufeld 1996; Bergin et al. 1998; Flower \& Pineau des Forêts 2010). The abundance of $\mathrm{H}_{2} \mathrm{O}$ in the hot gas is two orders of magnitude higher than that obtained for the warm gas, indicating that all of the available oxygen not locked in $\mathrm{CO}$ has been converted to $\mathrm{H}_{2} \mathrm{O}$. The derived $\mathrm{H}_{2} \mathrm{O}$ abundances are reported in Table 4.

The low water abundance associated with the warm gas component confirms previous findings in molecular outflows based on a limited number of lines (e.g., Bjerkeli et al. 2012; Vasta et al. 2012; Tafalla et al. 2013; Santangelo et al. 2013). Moreover, we also confirmed the presence of a hot gas component at higher abundance that so far has been clearly identified only for the B2 shock position of the L1448 outflow (Santangelo et al. 2013). The presence of warm and hot water components have been suggested by Goicoechea et al. (2012) and Dionatos et al. (2013) in shocks close to several Class 0 sources in Serpens. Therefore, our results confirm that in bow-shocks far from the driving source there is a bimodal distribution, which seems to be a common shock characteristic.

Nisini et al. (2010b) obtained the line cooling due to $\mathrm{H}_{2}$ in the B1 shock position, which is on the order ${ }^{5}$ of $0.03 L_{\odot}$. Here,

$5 L_{\mathrm{H}_{2}}$ has been corrected for a distance of $250 \mathrm{pc}$ while in Nisini et al. (2010b) the adopted distance is $440 \mathrm{pc}$. 
we estimated the total luminosity of water lines, $L_{\mathrm{H}_{2} \mathrm{O}}$, from the predicted line fluxes of the best-fit model shown in Fig. 8. We obtained $\sim 0.002 L_{\odot}$ and $\sim 0.03 L_{\odot}$ for the warm and the hot gas components, respectively. The derived $\mathrm{CO}$ luminosity of the warm component is $0.004 L_{\odot}$, hence the contribution of water to the line cooling is $50 \%$ of the CO luminosity. The luminosity of hot $\mathrm{CO}$ gas component, on the other hand, is $0.01 L_{\odot}$, and therefore the far-IR cooling of $\mathrm{H}_{2} \mathrm{O}$ dominates in front of $\mathrm{CO}$, and it contributes equally as the $\mathrm{H}_{2}$ line cooling. The results are summarized in Table 4.

Finally, we calculated the total far-IR cooling in the B1 shock region following the definition of Nisini et al. (2002), where $L_{\mathrm{FIR}}=L_{\mathrm{OI}}+L_{\mathrm{CO}}+L_{\mathrm{H}_{2} \mathrm{O}}+L_{\mathrm{OH}}$. Using the line fluxes reported by Benedettini et al. (2012), we estimated the total luminosity of [OI] and $\mathrm{OH}$, which are $L_{\mathrm{OI}} \simeq 2 \times 10^{-3} L_{\odot}$ and $L_{\mathrm{OH}} \simeq 4 \times 10^{-4} L_{\odot}$. For $\mathrm{CO}$ and $\mathrm{H}_{2} \mathrm{O}$ we considered the contribution of the two gas components. One can clearly see that the far-IR cooling is dominated by the contribution of $\mathrm{H}_{2} \mathrm{O}$ and $\mathrm{CO}$ lines, followed by [OI] and $\mathrm{OH}$. The total far-IR cooling estimated in $\mathrm{B} 1$ is $\sim 0.05 L_{\odot}$. It is worth noticing that the far-IR luminosity has been computed using similar beam sizes for all species, while the $\mathrm{H}_{2}$ luminosity was estimated with a smaller beam.

Shock models produce markedly different predictions on the $\mathrm{H}_{2} \mathrm{O}$ cooling function, depending on the nature of the shock, either C-type (MHD) or J-type shocks. We make here a simple comparison with the predictions from the steady-state shock models of Flower \& Pineau des Forêts (2010), for a shock propagating at $v=20 \mathrm{~km} \mathrm{~s}^{-1}$ into gas with pre-shock density of $10^{4} \mathrm{~cm}^{-3}$. Our goal is to identify qualitative trends of the properties of the shock responsible for the $\mathrm{H}_{2} \mathrm{O}$ emission detected. As pointed out by Gueth et al. (1996), the B1 bow-shock propagates into gas previously accelerated by the ejection associated with B2. Maximum velocities of 5-10 $\mathrm{km} \mathrm{s}^{-1}$ are reported in the B2 outflow cavity (Vasta et al. 2012). For this reason, we consider that velocities detected in the $\mathrm{H}_{2} \mathrm{O}$ gas towards $\mathrm{B} 1$ $\left(\approx 30 \mathrm{~km} \mathrm{~s}^{-1}\right.$; Fig. 4) are not inconsistent with a shock velocity of about $20 \mathrm{~km} \mathrm{~s}^{-1}$.

For the warm $\left(T_{\text {kin }} \simeq 250 \mathrm{~K}\right)$ gas component, the $\mathrm{H}_{2} \mathrm{O}$ line cooling is $\approx 1.5 \times 10^{-19} \mathrm{erg} \mathrm{cm}^{-3} \mathrm{~s}^{-1}$, in agreement with the value predicted in the molecular reformation zone of a J-type shock (see Fig. 2 in Flower \& Pineau des Forêts 2010; see also Benedettini et al. 2012). For the hot $\left(T_{\text {kin }} \simeq 1000 \mathrm{~K}\right)$ gas component, the $\mathrm{H}_{2} \mathrm{O}$ line cooling is $\approx 2 \times 10^{-16} \mathrm{erg} \mathrm{cm}^{-3} \mathrm{~s}^{-1}$, a value several orders of magnitude higher than that predicted by the $\mathrm{C}$-shock model, but well in the range of values expected in the J-type shock. Therefore, the simple comparison suggests that the hot gas layer is excited in a non-dissociative J-type shock.

\section{Summary and conclusions}

As part of the CHESS key program, we have analyzed the $\mathrm{H}_{2} \mathrm{O}$ emission towards the shock region L1157-B1. A grand total of $13 \mathrm{H}_{2} \mathrm{O}$ lines (both ortho and para) have been detected with HIFI and PACS instruments arising from transitions with rather low $E_{\mathrm{u}}$, from $26.7 \mathrm{~K}$ to $319.5 \mathrm{~K}$. The PACS and HIFI observations towards the L1157-B1 bow-shock have revealed the presence of several gas components with different excitation. Our main conclusions can be summarized as follows:

1. The bulk of $\mathrm{H}_{2} \mathrm{O}$ emission originates in the $\mathrm{B} 1$ bow-shock.

2. An absorption feature is detected in the line profiles that are connected with the ground state level. It arises from a water-enriched layer $\left(X\left[\mathrm{H}_{2} \mathrm{O}\right] \simeq 10^{-7}\right)$ at the surface of the cloud formed as a result of water ice photodesorption from interstellar grain mantles, driven by the external UV photons due to the interstellar radiation field.

3. The LVG analysis of the $\mathrm{H}_{2} \mathrm{O}$ emission associated with the bright high-excitation region (i.e., the bow-shock) has permitted us to identify two physical components. A warm, dense gas $\left(T_{\text {kin }} \sim 200-300 \mathrm{~K}, n\left(\mathrm{H}_{2}\right) \simeq(1-3) \times 10^{6} \mathrm{~cm}^{-3}\right)$ component traced mainly by the low-excitation lines of water, with an assumed extent of $10^{\prime \prime}$. The OPR- $\mathrm{H}_{2}$ in the warm gas is $\simeq 0.5$. The hot $\left(T_{\text {kin }} \simeq 1000 \mathrm{~K}\right)$ component is made of tenuous gas at a much lower density (a few $10^{3-4} \mathrm{~cm}^{-3}$ ) similar to that of the parental cloud. It is much more compact, with a typical size of $2^{\prime \prime}-5^{\prime \prime}$. The OPR- $\mathrm{H}_{2}$ in the warm gas is $\simeq 3.0$, equal to its value at LTE.

4. These two shock components present marked differences in terms of water enrichment. While the derived abundance in the warm gas is $(0.7-2) \times 10^{-6}$, the water abundance estimated in the hot gas is much higher, around (1.2-3.6) $\times$ $10^{-4}$, indicating that all available oxygen not locked in CO is driven into $\mathrm{H}_{2} \mathrm{O}$. The far-IR cooling of the bow-shock appears to be equally dominated by both $\mathrm{H}_{2}$ and the hot water component.

5. A simple comparison of the water line cooling properties with the steady-state shock models of Flower \& Pineau des Forêts (2010) is consistent with a J-type shock origin for both components. The exact nature of the hot water spot and its relation with the jet impact against the cavity remains to be established. The low density of the hot $\mathrm{H}_{2} \mathrm{O}$ gas suggests that the shock propagates into a region of much lower density, either in the ambient cloud or the outflow cavity gas.

Higher-angular resolution observations are needed to understand the structure of the L1157-B1 bow-shock region. We expect that a detailed, multiline study and comparison of the emission properties of the major cooling agents, $\mathrm{CO}, \mathrm{H}_{2} \mathrm{O}$ and $\mathrm{H}_{2}$ at infrared wavelengths with shock model predictions, will help us to clarify the origin of the different shock components revealed by PACS and HIFI and the relation they hold to each other (Cabrit et al., in prep.).

Acknowledgements. The authors are grateful to the anonymous referee and the editor, Dr. Malcolm Walmsley, for valuable comments. G.B. is grateful to $\mathbf{M}$. Pereira-Santaella for fruitful discussion. G. Busquet, M. Benedettini, C. Codella, B. Nisini, A. I. Gómez-Ruiz, and A. M. di Giorgio are supported by the Italian Space Agency (ASI) project I/005/11/0. B. Lefloch thanks the Spanish MEC for funding support through grant SAB2009-0011. B. Lefloch, C. Ceccarelli, and L. Wiesenfeld acknowledge funding from the French Space Agency CNES and the National Research Agency funded project FORCOM, ANR-08-BLAN0225. S. Viti acknowledges support from the [European Community's] Seventh Framework Programme [FP7/2007-2013] under grant agreement $n^{\circ} 238258$. A. Gusdorf acknowledges support by grant ANR-09-BLAN-0231-01 from the French Agence Nationale de la Recherche as part of the SCHISM project. HIFI has been designed and built by a consortium of institutes and university departments from across Europe, Canada and the United States under the leadership of SRON Netherlands Institute for Space Research, Groningen, The Netherlands, and with major contributions from Germany, France and the US. Consortium members are: Canada: CSA, U.Waterloo; France: CESR, LAB, LERMA, IRAM; Germany: KOSMA, MPIfR, MPS; Ireland, NUI Maynooth; Italy: ASI, IFSI-INAF, Osservatorio Astrofisico di Arcetri-INAF; Netherlands: SRON, TUD; Poland: CAMK, CBK; Spain: Observatorio Astronómico Nacional (IGN), Centro de Astrobiología (CSIC-INTA). Sweden: Chalmers University of Technology - MC2, RSS \& GARD; Onsala Space Observatory; Swedish National Space Board, Stockholm University - Stockholm Observatory; Switzerland: ETH Zurich, FHNW; USA: Caltech, JPL, NHSC. PACS has been developed by a consortium of institutes led by MPE (Germany) and including UVIE (Austria); KU Leuven, CSL, IMEC (Belgium); CEA, LAM (France); MPIA (Germany); INAF-IFSI/OAA/OAP/OAT, LENS, SISSA (Italy); IAC (Spain). This development has been supported by the funding agencies BMVIT (Austria), ESA-PRODEX (Belgium), CEA/CNES (France), DLR (Germany), ASI/INAF (Italy), and CICYT/MCYT (Spain). 


\section{References}

Arce, H. G., Santiago-García, J., Jørgensen, J. K., Tafalla, M., \& Bachiller, R. 2008, ApJ, 681, L21

Bachiller, R. 1996, ARA\&A, 34, 111

Bachiller, R., \& Pérez Gutiérrez, M. 1997, ApJ, 487, L93

Bachiller, R., Pérez Gutiérrez, M., Kumar, M. S. N., \& Tafalla, M. 2001, A\&A, 372, 899

Benedettini, M., Viti, S., Codella, C., et al. 2007, MNRAS, 381, 1127

Benedettini, M., Busquet, G., Lefloch, B., et al. 2012, A\&A, 539, L3

Benedettini, M., Viti, S., Codella, C., et al. 2013, MNRAS, 436, 179

Bergin, E. A., Neufeld, D. A., \& Melnick, G. J. 1998, ApJ, 499, 777

Bjerkeli, P., Liseau, R., Olberg, M., et al. 2009, A\&A, 507, 1455

Bjerkeli, P., Liseau, R., Larsson, B., et al. 2012, A\&A, 546, A29

Bjerkeli, P., Liseau, R., Nisini, B., et al. 2013, A\&A, 552, L8

Caratti o Garatti, A., Giannini, T., Nisini, B., \& Lorenzetti, D. 2006, A\&A, 449, 1077

Ceccarelli, C., Maret, S., Tielens, A. G. G. M., Castets, A., \& Caux, E. 2003, A\&A, 410, 587

Ceccarelli, C., Bacmann, A., Boogert, A., et al. 2010, A\&A, 521, L22

Codella, C., Benedettini, M., Beltrán, M. T., et al. 2009, A\&A, 507, L25

Codella, C., Lefloch, B., Ceccarelli, C., et al. 2010, A\&A, 518, L112

Codella, C., Ceccarelli, C., Lefloch, B., et al. 2012, ApJ, 757, L9

Coutens, A., Vastel, C., Caux, E., et al. 2012, A\&A, 539, A132

Daniel, F., Dubernet, M.-L., Pacaud, F., \& Grosjean, A. 2010, A\&A, 517, A13

Daniel, F., Dubernet, M.-L., \& Grosjean, A. 2011, A\&A, 536, A76

Davis, C. J., \& Eisloeffel, J. 1995, A\&A, 300, 851

de Graauw, T., Helmich, F. P., Phillips, T. G., et al. 2010, A\&A, 518, L6

Dionatos, O., Jørgensen, J. K., Green, J. D., et al. 2013, A\&A, 558, A88

Draine, B. T., Roberge, W. G., \& Dalgarno, A. 1983, ApJ, 264, 485

Dubernet, M.-L., Grosjean, A., Flower, D., et al. 2006, Journal of Plasma Research SERIES, 7, 356

Dubernet, M.-L., Daniel, F., Grosjean, A., \& Lin, C. Y. 2009, A\&A, 497, 911

Dubernet, M.-L., Alexander, M. H., Ba, Y. A., et al. 2013, A\&A, 553, A50

Flower, D. R., \& Pineau des Forêts, G. 2010, MNRAS, 406, 1745

Flower, D. R., \& Pineau des Forêts, G. 2012, MNRAS, 421, 2786

Franklin, J., Snell, R. L., Kaufman, M. J., et al. 2008, ApJ, 674, 1015

Frerking, M. A., Langer, W. D., \& Wilson, R. W. 1982, ApJ, 262, 590

Giannini, T., Nisini, B., \& Lorenzetti, D. 2001, ApJ, 555, 40
Goicoechea, J. R., Cernicharo, J., Karska, A., et al. 2012, A\&A, 548, A77

Gueth, F., Guilloteau, S., \& Bachiller, R. 1996, A\&A, 307, 891

Gueth, F., Guilloteau, S., \& Bachiller, R. 1998, A\&A, 333, 287

Gusdorf, A., Cabrit, S., Flower, D. R., \& Pineau des Forêts, G. 2008a, A\&A, 482, 809

Gusdorf, A., Pineau des Forêts, G., Cabrit, S., \& Flower, D. R. 2008b, A\&A, 490, 695

Hollenbach, D., \& McKee, C. F. 1989, ApJ, 342, 306

Hollenbach, D., Kaufman, M. J., Bergin, E. A., \& Melnick, G. J. 2009, ApJ, 690, 1497

Kaufman, M. J., \& Neufeld, D. A. 1996, ApJ, 456, 611

Lefloch, B., Cernicharo, J., Cabrit, S., et al. 2003, ApJ, 590, L41

Lefloch, B., Cabrit, S., Busquet, G., et al. 2012, ApJ, 757, L25

Looney, L. W., Tobin, J. J., \& Kwon, W. 2007, ApJ, 670, L131

Maret, S., Bergin, E. A., Neufeld, D. A., et al. 2009, ApJ, 698, 1244

Neufeld, D. A., Melnick, G. J., \& Harwit, M. 1998, ApJ, 506, L75

Neufeld, D. A., Melnick, G. J., Sonnentrucker, P., et al. 2006, ApJ, 649, 816

Neufeld, D. A., Nisini, B., Giannini, T., et al. 2009, ApJ, 706, 170

Nisini, B., Giannini, T., \& Lorenzetti, D. 2002, ApJ, 574, 246

Nisini, B., Codella, C., Giannini, T., et al. 2007, A\&A, 462, 163

Nisini, B., Benedettini, M., Codella, C., et al. 2010a, A\&A, 518, L120

Nisini, B., Giannini, T., Neufeld, D. A., et al. 2010b, ApJ, 724, 69

Ott, S. 2010, in Astronomical Data Analysis Software and Systems XIX, eds. Y. Mizumoto, K.-I. Morita, \& M. Ohishi, ASP Conf. Ser., 434, 139

Pickett, H. M., Poynter, R. L., Cohen, E. A., et al. 1998, J. Quant. Spectr. Rad. Transf., 60, 883

Pilbratt, G. L., Riedinger, J. R., Passvogel, T., et al. 2010, A\&A, 518, L1

Poglitsch, A., Waelkens, C., Geis, N., et al. 2010, A\&A, 518, L2

Roelfsema, P. R., Helmich, F. P., Teyssier, D., et al. 2012, A\&A, 537, A17

Santangelo, G., Nisini, B., Antoniucci, S., et al. 2013, A\&A, 557, A22

Tafalla, M., \& Bachiller, R. 1995, ApJ, 443, L37

Tafalla, M., Liseau, R., Nisini, B., et al. 2013, A\&A, 551, A116

Takami, M., Karr, J. L., Nisini, B., \& Ray, T. P. 2011, ApJ, 743, 193

van Dishoeck, E. F., Kristensen, L. E., Benz, A. O., et al. 2011, PASP, 123, 138

Vasta, M., Codella, C., Lorenzani, A., et al. 2012, A\&A, 537, A98

Wilgenbus, D., Cabrit, S., Pineau des Forêts, G., \& Flower, D. R. 2000, A\&A, 356,1010

Zhang, Q., Ho, P. T. P., \& Wright, M. C. H. 2000, AJ, 119, 1345 